\title{
The importance of Thermo-Hydro-Mechanical couplings and microstructure to strain localization in 3D continua with application to seismic faults. Part I: Theory and Linear Stability Analysis
}

\author{
Hadrien Rattez ${ }^{\mathrm{a}}$, Ioannis Stefanou ${ }^{\mathrm{a}, *}$, Jean Sulem $^{\mathrm{a}}$ \\ ${ }^{a}$ Laboratoire Navier, UMR 8205, École des Ponts ParisTech, IFSTTAR, CNRS, \\ Champs-sur-Marne, France
}

\begin{abstract}
A Thermo-Hydro-Mechanical (THM) model for Cosserat continua is developed to explore the influence of frictional heating and thermal pore fluid pressurization on the strain localization phenomenon. A general framework is presented to conduct a bifurcation analysis for elasto-plastic Cosserat continua with THM couplings and predict the onset of instability. Furthermore, the presence of an internal length in Cosserat continua enables to estimate the thickness of the localization zone. This is done by performing a linear stability analysis of the system and looking for the selected wavelength corresponding to the instability mode with fastest finite growth coefficient. These concepts are applied to the study of fault zones under fast shearing. For doing so, we consider a model of a sheared saturated infinite granular layer. The influence of THM couplings on the bifurcation state and the shear band width is investigated. Taking representative parameters for a centroidal fault gouge, the evolution of the thickness of the localized zone under continuous shear is studied. Furthermore, the effect of grain crushing inside the shear band is explored by varying the internal length of the constitutive law.
\end{abstract}

Keywords: Cosserat continuum, Linear stability analysis,

\footnotetext{
${ }^{*}$ Corresponding author

Email address: ioannis.stefanou@enpc.fr (Ioannis Stefanou)
} 
Thermo-Hydro-Mechanical couplings, Strain localization, Fault gouge

\section{Introduction}

Strain localization is of major importance in fault zones as it affects shear heating and pore pressure build up during seismic slip (Kanamori and Brodsky, 2004). In this paper we investigate the effect of microstructure and of various

5 Thermo-Hydro-Mechanical (THM) couplings on the behavior of geomaterials and, in particular, on the behavior of mature faults zones during pre- and coseismic slip. Seismic slip is accompanied by extreme shear strain localization into a narrow, thin zone, which is commonly called Principal Slip Zone (PSZ). According to field observations, the PSZ has a finite thickness (see for instance 10 Punchbowl fault, San Andreas system (Chester and Chester, 1998), Big Hole normal fault, Utah (Shipton et al., 2006), Median Tectonic line, Japan (Wibberley and Shimamoto, 2003)), which varies from hundreds of microns to few centimetres (Sibson, 2003), depending on the size of the microstructure and of THM mechanisms. The PSZ lies within a zone of highly fragmented, granulated 15 material called fault gouge (Ben-Zion and Sammis, 2003).

Theoretical and experimental research show that strain localization is caused and enhanced by weakening mechanisms that can either be of pure mechanical origin (e.g. geometrical and mechanical changes of the solid skeleton (Togo and Shimamoto, 2012), such as grain cataclasis, reorientation, debonding etc.) or 20 of a combination of various physico-chemical couplings (Sulem and Stefanou, 2016). For instance, thermal pressurization of the pore fluid in saturated fault materials is a THM mechanism that plays a fundamental role in the weakening of fault zones (Lachenbruch, 1980, Viesca and Garagash, 2015). Thermal pressurization is a consequence of the contrast between the thermal expansion coefficient of the pore fluids and the solid matrix (Rice, 2006) and leads to a decrease of the effective mean stress and consequently to a reduction of the shear strength of the gouge. The thickness of the PSZ governs the temperature build-up and the overall energy budget. It is worth mentioning that the acti- 
vation of other multi-physical phenomena that involve chemical processes are also controlled by the thickness of the localization zone (Brantut et al., 2011, Veveakis et al., 2013, Platt et al., 2015, Sulem and Stefanou, 2016).

Models that are able to describe the localization thickness and its evolution have to take into account both the size of the microstructure of a fault gouge as well as the multi-physical couplings that take place during seismic slip. Cosserat theory allows in a natural way to account for the aforementioned characteristics, leading to a shear band of finite thickness even under low strain rates (Mühlhaus and Vardoulakis, 1987). The use of this theoretical framework is also justified by the fact that it can cover a large spectrum of strain rates, i.e. from very low (pre-seismic) to quite high (co-seismic). Notice that existing models for

40 fault gouges based on the classical, Cauchy continuum (also called Boltzmann continuum (Vardoulakis, 2009)) lead to an infinitely small localized zone (slip on a mathematical plane) (Vardoulakis, 1985) unless rate-dependent constitutive behavior is considered for high strain rates and/or THM couplings are explicitly taken into account (Rice et al., 2014, Platt et al., 2014). Moreover, grain size cannot be considered in the constitutive description of Cauchy continua, despite the fact that it has been recognized to play an important role on fault gouge behavior (Anthony and Marone, 2005, Cashman et al., 2007, Phillips and White, 2017).

Cosserat continuum (Cosserat and Cosserat, 1909) is a special case of micromorphic continua (Germain, 1973, Godio et al., 2016), also called generalized or higher order continua. In addition to the translational degrees of freedom of the Cauchy continuum, Cosserat theory considers rotational degrees of freedom at the material point that allow for a better representation of the physics and the mechanical behavior of heterogeneous solids with non-negligible microstructure. Cosserat continuum theory naturally incorporates one or several material lengths related to the microstructure in the constitutive equations of the material (see Appendix B).

Cosserat continuum has been previously used for studying the behavior of fault gouges and strain localization (Sulem et al., 2011, Veveakis et al., 2013). In 
these works the conditions for the onset of localization were investigated under THM couplings with a microstructure of given size. In the present papers (Part I and II) we extend the aforementioned works by studying (a) the evolution of the localization zone thickness and its dependency on various parameters such the size of the microstructure, (b) the full stress-strain response of the fault 65 gouge, which is related to the transition from seismic to aseismic slip (Scholz, 2002, Tse and Rice, 1986) and (c) the apparent rate dependency of the system due to THM couplings even under rate-independent constitutive laws.

In part I, we focus mainly on point (a) using bifurcation theory and Linear Stability Analysis (LSA). The approach is analytical and it allows to explore qualitatively the influence of the evolution of the hardening parameter and of the grain size on the thickness of the localized zone. In Sections 2 and 3 we present the momentum, mass and energy balance equations and the full constitutive equations for general Cosserat elasto-plastic continua. The bifurcation analysis in this framework is presented in Section 4 and linked with classical results

75 like the singularity of the acoustic tensor. Finally, the bifurcation analysis is applied to the problem of slip in a fault zone (Section 5) and the influence of the main parameters of the model is investigated as far it concerns the onset of localization and the shear band thickness evolution.

\section{Basic concepts of three-dimensional Cosserat continuum mechan- ics and balance equations}

The Cosserat continuum is a special case of first order micromorphic continua, for which the particle is considered rigid (Godio et al., 2016, Stefanou et al., 2010). In the frame of Cosserat theory the kinematics of a material point in three-dimensional (3D) space is described by six degrees of freedom, which are three translations $u_{i}$ and three rotations $\omega_{i}^{c}(i=1,2,3)$. In this section, the basic concepts of Cosserat theory are outlined. 


\subsection{Cosserat kinematics}

Compared to a Cauchy continuum formed by a set of particles identified by their coordinates $x_{i}$, we attach to every particle a system of axes parallel to the Cartesian one and with $\mathrm{M}$, the center of mass of the particle, as origin.

If we consider a point $\mathrm{M}$ ', in the particle of center M, defined by its coordinates $x_{i}^{\prime}$, the displacement field in $\mathrm{M}^{\prime}, u_{i}^{\prime}$, can be written as follows, considering only terms of first order.

$$
u_{i}^{\prime}=u_{i}+\chi_{i j} x_{j}^{\prime}
$$

Einstein summation convention is followed herein. $\chi_{i j}$ is the micro-deformation tensor. As the microstructure is considered rigid in Cosserat theory, the microvolume cannot deform and can only rotate. Thus, the micro-deformation tensor $\chi_{i j}$ is antisymmetric and is called the Cosserat rotation $\omega_{i j}^{c}$. As $\omega_{i j}^{c}$ is antisymmetric, we can write :

$$
\omega_{i j}^{c}=-e_{i j k} \omega_{k}^{c}
$$

where $e_{i j k}$ is the Levi-Civita symbol.

The following kinematic fields are introduced: the deformation tensor $\gamma_{i j}$ - which is split into its symmetric $\varepsilon_{i j}$ and antisymmetric part $\gamma_{[i j]}$ - and the curvature tensor $\kappa_{i j}$ - also split into its symmetric $\kappa_{(i j)}$ and antisymmetric part $\kappa_{[i j]}$.

$$
\begin{array}{r}
\varepsilon_{i j}=\frac{1}{2}\left(u_{i, j}+u_{j, i}\right) \quad \Omega_{i j}=\frac{1}{2}\left(u_{i, j}-u_{j, i}\right) \\
\gamma_{[i j]}=\Omega_{i j}-\omega_{i j}^{c} \quad \kappa_{i j}=\omega_{i, j}^{c} \\
\gamma_{i j}=\varepsilon_{i j}+\gamma_{[i j]}=u_{i, j}-\omega_{i j}^{c}=u_{i, j}+e_{i j k} \omega_{k}^{c}
\end{array}
$$

The macroscopic strain and rotation tensors $\left(\varepsilon_{i j}\right.$ and $\left.\Omega_{i j}\right)$ are the symmetric and antisymmetric parts of the displacement gradient as in a classical Cauchy 
continuum. $\gamma_{[i j]}$ is the difference between the macroscopic rotation $\Omega_{i j}$ and the rotation of the microstructure $\omega_{i j}^{c}$. The curvature tensor $\kappa_{i j}$ is defined as the gradient of Cosserat rotations.

\subsection{Momentum and angular momentum balance equations}

Correspondingly the stress tensor $\tau_{i j}$ is also divided into its symmetric $\sigma_{i j}$ and antisymmetric part $\tau_{[i j]}$. The symmetric part corresponds to the macroscopic stresses (the ones that are considered in Cauchy continuum), the antisymmetric one is nonzero in general. A tensor for the couple-stress is defined by $\mu_{i j}$ linked to the curvature.
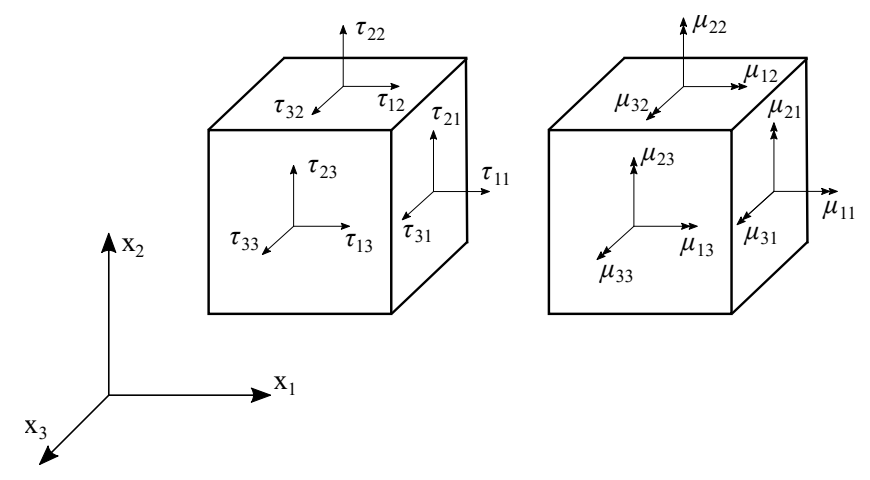

Figure 1: Representation of stress and couple-stress components.

The momentum balance equations can be written as follows (assuming no body forces acting on the medium)(Vardoulakis and Sulem, 1995, Stefanou et al., 2017):

$$
\begin{array}{r}
\tau_{i j, j}-\rho \frac{\partial^{2} u_{i}}{\partial t^{2}}=0 \\
\mu_{i j, j}-e_{i j k} \tau_{j k}-\rho I \frac{\partial^{2} \omega_{i}^{c}}{\partial t^{2}}=0
\end{array}
$$

where $\rho$ is the density, $I$ is the micro-inertia, which is considered isotropic here. For example, $I=\frac{2}{5} R^{2}$ if we identify the particle as spherical grains with ${ }_{120}$ radius $\mathrm{R}\left(I=\frac{1}{2} R^{2}\right.$ in $\left.2 \mathrm{D}\right)$ (Vardoulakis and Sulem, 1995). 


\subsection{Energy balance equation}

The energy and mass balance equations as classically derived for a saturated Cauchy continuum are extended here to a saturated Cosserat continuum.

The energy conservation in a quasi-static transformation is expressed as follows:

$$
\rho C \frac{\partial \mathrm{T}}{\partial t}=P_{H}-j_{i, i}^{Q}
$$

where $\rho C$ is the specific heat per unit volume of the material in its reference state and $j_{i}^{Q}$ represents the heat flux density. It is assumed here that the rate of heat produced $P_{H}$ is due to plastic dissipation, thus, $P_{H}=\sigma_{i j} \dot{\varepsilon}_{i j}^{p}+\tau_{[i j]} \dot{\gamma}_{[i j]}^{p}+\mu_{i j} \dot{\kappa}_{i j}^{p}$. This expression is the sum of three terms, one corresponding to the plastic work due to the symmetric part of the stress tensor (Cauchy), $\sigma_{i j} \dot{\varepsilon}_{i j}^{p}$, and the two others are due to Cosserat effects (Vardoulakis and Sulem, 1995). $\dot{\varepsilon}_{i j}^{p}$, $\dot{\gamma}_{[i j]}^{p}$ and ${\dot{\kappa_{i j}}}^{p}$ are the plastic symmetric deformation rate, plastic antisymmetric deformation rate and the plastic curvature rate tensors respectively. The heat flux is linked to the temperature gradient by Fourier's law:

$$
j_{i}^{Q}=-k_{T} T_{, i}
$$

where $k_{T}$ is the thermal conductivity of the material which is assumed homogeneous here. Substituting these two expressions gives the energy balance equation.

$$
\rho \mathrm{C}\left(\frac{\partial \mathrm{T}}{\partial t}-c_{t h} T_{, i i}\right)=\sigma_{i j} \dot{\varepsilon}_{i j}^{p}+\tau_{[i j]} \dot{\gamma}_{[i j]}^{p}+\mu_{i j} \dot{\kappa}_{i j}^{p}
$$

where $c_{t h}=\frac{k_{T}}{\rho C}$ is the thermal diffusivity.

\subsection{Mass balance equation}

${ }_{140}$ We consider a porous medium consisting of two phases, i.e. the solid skeleton and the fluid contained in the pores. The conservation of the mass of the skeleton and the fluid, when no mass exchange occurs is: 


$$
\begin{aligned}
\frac{d^{s} m^{s}}{d t} & =0 \\
\frac{d^{f} m^{f}}{d t} & =0
\end{aligned}
$$

where $\frac{d^{\pi}}{d t}$ refers to the particle derivative with respect to particle $\pi(=s$ or $f$ ) (Coussy, 2004). $m^{f}$ and $m^{s}$ are respectively the fluid mass and the skeleton mass per unit volume of the medium. If $\rho_{s}$ and $\rho_{f}$ are the skeleton and fluid mass densities so that $\rho_{s}(1-n) d \Omega^{t}$ and $\rho_{f} n d \Omega^{t}$ are respectively the skeleton mass and the fluid mass currently contained in the material volume with an Eulerian porosity $n$, we obtain:

$$
\begin{array}{r}
\frac{d^{s} m^{s}}{d t}=\frac{d^{s}\left(\rho^{s}(1-n) d \Omega^{t}\right)}{d t}=\frac{d^{s}\left(\rho^{s}(1-n)\right)}{d t} d \Omega^{t}+\rho^{s}(1-n) \frac{d^{s} d \Omega^{t}}{d t} \\
\frac{d^{f} m^{f}}{d t}=\frac{d^{f}\left(\rho^{f} n d \Omega^{t}\right)}{d t}=\frac{d^{f}\left(\rho^{f} n\right)}{d t} d \Omega^{t}+\rho^{f} n \frac{d^{f} d \Omega^{t}}{d t}
\end{array}
$$

By applying the Eulerian continuity conditions and using the expression of the particle derivative (Coussy, 2004) we get:

$$
\begin{aligned}
\frac{\partial\left(\rho^{s}(1-n)\right)}{\partial t}+\left(\left(\rho^{s}(1-n)\right) V_{i}^{s}\right)_{, i} & =0 \\
\frac{\partial\left(\rho^{f} n\right)}{\partial t}+\left(\rho^{f} n V_{i}^{f}\right)_{, i} & =0
\end{aligned}
$$

where $V_{i}^{s}$ and $V_{i}^{f}$ are the velocity fields of the solid and fluid phase respectively. ()$_{, i}$ represents the divergence operator applied to the current state. However, we keep a small strain framework and therefore no distinction will be made in the following between the reference and the deformed configuration. becomes

$$
\begin{array}{r}
-\rho^{s} \frac{\partial n}{\partial t}+(1-n) \frac{\partial \rho^{s}}{\partial t}+\rho^{s}\left((1-n) V_{i}^{s}\right)_{, i}=0 \\
\frac{n}{\rho^{f}} \frac{\partial \rho^{f}}{\partial t}+\frac{\partial n}{\partial t}+n V_{i, i}^{f}=0
\end{array}
$$


Finally, adding Eq. 2.12 divided by $\rho^{s}$ and Eq. 2.13 yields:

$$
\frac{n}{\rho^{f}} \frac{\partial \rho^{f}}{\partial t}+\frac{1-n}{\rho^{s}} \frac{\partial \rho^{s}}{\partial t}+V_{i, i}^{s}+\left(n\left(V_{i}^{f}-V_{i}^{s}\right)\right)_{, i}=0
$$

If we assume that the density of the fluid $\rho^{f}$ and of the solid $\rho^{s}$ only depend on the pressure and temperature (Rice, 2006), we obtain:

$$
\begin{gathered}
\frac{\partial \rho^{f}}{\partial t}=\rho^{f} \beta^{f} \frac{\partial p}{\partial t}-\rho^{f} \lambda^{f} \frac{\partial T}{\partial t} \\
\frac{\partial \rho^{s}}{\partial t}=\rho^{s} \beta^{s} \frac{\partial p}{\partial t}-\rho^{s} \lambda^{s} \frac{\partial T}{\partial t}
\end{gathered}
$$

160 and the solid phase respectively and $\lambda^{f}, \lambda^{s}$ the thermal expansivities per unit volume. The fluid mass flux is assumed to obey the isotropic Darcy's law (for a quasi-static flow without any body force).

$$
n\left(V_{i}^{f}-V_{i}^{s}\right)=-\frac{\chi}{\eta^{f}} p_{, i}
$$

where $\chi$ is the intrinsic permeability of the porous medium, and $\eta^{f}$ is the viscosity of the pore fluid. Inserting Eqs. 2.15, 2.16 in Eq. 2.14 we obtain

$$
\frac{\partial p}{\partial t}=c_{h y} p_{, i i}+\frac{\lambda^{*}}{\beta^{*}} \frac{\partial \mathrm{T}}{\partial t}-\frac{1}{\beta^{*}} \frac{\partial \varepsilon_{v}}{\partial t}
$$

where $c_{h y}=\chi /\left(\eta^{f} \beta^{*}\right)$ is the hydraulic diffusivity, $\beta^{*}=n \beta^{f}+(1-n) \beta^{s}$ is the mixture compressibility, $\lambda^{*}=\left(n \lambda^{f}+(1-n) \lambda^{s}\right)$ is the coefficient of thermal expansion of the soil-water mixture (Vardoulakis, 1986). This formulation differs from Sulem et al. (2011) and Rice (2006), where the authors introduce the mechanical constitutive equation through the variation of porosity. Therefore, instead of having the term depending on total volumetric deformation $\varepsilon_{v}$, they have one depending on plastic volumetric deformation $\varepsilon_{v}^{p}$. In Eq. 2.17, we keep the volumetric deformation without assuming any particular constitutive equation (Lachenbruch, 1980). Its evolution can be controlled by damage, plastic 
lations in the case of an elasto-plastic constitutive model is shown in Appendix

\section{Thermo-Hydro elasto-plastic model for fluid-saturated isotropic Cosserat materials}

For simplicity, constitutive equations are written in terms of the Terzaghi effective stress for both the elastic and plastic strains. However the underlying assumption of elastically incompressible grains can be overcome without difficulty by resorting to Biot's theory of poromechanics (Biot and Willis, 1957).

\subsection{Isotropic elastic constitutive law}

The general constitutive equations for a linear isotropic elastic Cosserat continuum are defined by six coefficients. The two classical deformation moduli, K and $\mathrm{G}$, and four additional coefficients, $G_{c}, L, M$, and $M_{c}$ (Mindlin, 1964).

$$
\begin{array}{r}
\tau_{i j}=K \gamma_{k k}^{e} \delta_{i j}+2 G\left(\varepsilon_{i j}^{e}-\frac{1}{3} \gamma_{k k}^{e}\right)+2 G_{c} \gamma_{[i j]}^{e} \\
\mu_{i j}=L \kappa_{k k}^{e} \delta_{i j}+2 M\left(\kappa_{(i j)}^{e}-\frac{1}{3} \kappa_{k k}^{e}\right)+2 M_{c} \kappa_{[i j]}^{e}
\end{array}
$$

$(.)^{e}$ denotes elastic quantities. In comparison with the classical Cauchy continuum four additional moduli are used for an isotropic, centrosymmetric linear elastic Cosserat continuum. The first one is $G_{c}$, which has a dimension of stress and relates the antisymmetric parts of the stress and deformation tensors, which are conjugate in energy. The other moduli, $L, M$ and $M_{c}$ have the dimension of length squared times stress. Any ratio of $L, M$ or $M c$ to $K, G$ or $G_{c}$ results in a material parameter of dimension of length squared (Cowin, 1970).

In the analyses of simple shearing of an infinite layer presented in Section 5 the choice of $L$ does not have any influence due to invariance in $x_{1}$ and $x_{3}$ directions. Moreover, setting $M_{c}=M=\frac{G R^{2}}{h_{3}}$, where $h_{3}$ a coefficient defined in 
Appendix B, we assure that no out-of-plane moments are developed (see Fig. 1 and Eq. 3.2).

The generalized elastic stress-strain relationships are written as:

$$
\begin{gathered}
\tau_{i j}=C_{i j k l}^{e} \gamma_{k l} \\
\mu_{i j}=M_{i j k l}^{e} \kappa_{k l}
\end{gathered}
$$

where the elastic stiffness tensors $C_{i j k l}^{e}$ and $M_{i j k l}^{e}$ are derived from (Eqs. 3.1 $3.2)$.

$$
\begin{gathered}
C_{i j k l}^{e}=\left(K-\frac{2}{3} G\right) \delta_{i j} \delta_{k l}+\left(G+G_{c}\right) \delta_{i k} \delta_{j l}+\left(G-G_{c}\right) \delta_{i l} \delta_{j k} \\
M_{i j k l}^{e}=\left(L-\frac{2}{3} M\right) \delta_{i j} \delta_{k l}+\left(M+M_{c}\right) \delta_{i k} \delta_{j l}+\left(M-M_{c}\right) \delta_{i l} \delta_{j k}
\end{gathered}
$$

\subsection{Thermo-elasto-plastic incremental constitutive relationship}

Following Mühlhaus and Vardoulakis (1987), a flow theory of plasticity for granular media with Cosserat microstructure can be derived by keeping the same definitions for the yield surface and the plastic potential as in the classical theory and by generalizing the stress and strain invariants for Cosserat continua. We decompose the deformation rate tensor and the curvature rate tensor into elastic, plastic and thermal parts (Lemaitre et al., 2009):

$$
\dot{\gamma}_{i j}=\dot{\gamma}_{i j}^{e}+\dot{\gamma}_{i j}^{p}+\dot{\gamma}_{i j}^{t h} \quad \text { and } \quad \dot{\kappa}_{i j}=\dot{\kappa}_{i j}^{e}+\dot{\kappa}_{i j}^{p}
$$

(.) denotes the time derivative. Thermal strain rates are written as: $\dot{\gamma}_{i j}^{\text {th }}=$ $\alpha \dot{T} \delta_{i j}$ where $\alpha$ is the coefficient of thermal expansion. No thermal part for the curvature is considered as the thermal expansion for isotropic solids does not have a direct effect on the rotations inside the medium.

Denoting $F$ the yield function and assuming that $F$ depends on the stress invariants and on accumulated plastic strains $F=F\left(\tau, \sigma, \gamma^{p}, \varepsilon_{v}^{p}\right)$, we obtain

$$
\dot{F}=\frac{\partial F}{\partial \tau} \dot{\tau}+\frac{\partial F}{\partial \sigma} \dot{\sigma}+\frac{\partial F}{\partial \gamma^{p}} \dot{\gamma}^{p}+\frac{\partial F}{\partial \varepsilon_{v}^{p}}{\dot{\varepsilon_{v}}}^{p}=0
$$


An example of generalized invariants used for Cosserat media is presented in detail in part 4.

Denoting Q the plastic potential and $\dot{\lambda}$ the plastic multiplier, we have

$$
\begin{aligned}
& \dot{\gamma}_{i j}^{p}=\dot{\lambda} \frac{\partial Q}{\partial \tau_{i j}} \\
& \dot{\kappa}_{i j}^{p}=\dot{\lambda} \frac{\partial Q}{\partial \mu_{i j}}
\end{aligned}
$$

The hardening modulus $H_{s}$ is defined by:

$$
H_{s}=-\frac{\partial F}{\partial \gamma^{p}}
$$

We assume the following equalities that can be proven for several yield functions that have a linear dependence in $\tau$ and $\sigma$ (see for example Drucker-Prager in the following):

$$
\dot{\lambda}=\dot{\gamma}^{p} \quad \text { and } \quad \dot{\varepsilon}_{v}^{p}=\beta \dot{\gamma}^{p}
$$

The consistency condition gives

$$
\dot{\lambda}=\frac{<1>}{H_{p}}\left(\frac{\partial F}{\partial \tau_{i j}} C_{i j k l}^{e}\left(\dot{\gamma}_{k l}-\alpha \dot{T} \delta_{k l}\right)\right)+\frac{\partial F}{\partial \mu_{i j}} M_{i j k l}^{e} \dot{\kappa}_{k l}
$$

or,

$$
\dot{\lambda}=\frac{<1>}{H_{p}}\left(b_{k l}^{F}\left(\dot{\gamma}_{k l}-\alpha \dot{T} \delta_{k l}\right)+b_{k l}^{F M} \dot{\kappa}_{k l}\right)
$$

with

$$
\begin{array}{r}
H_{p}=\frac{\partial F}{\partial \tau_{i j}} C_{i j k l}^{e} \frac{\partial Q}{\partial \tau_{k l}}+\frac{\partial F}{\partial \mu_{i j}} M_{i j k l}^{e} \frac{\partial Q}{\partial \mu_{k l}}+H_{s} \\
<1>= \begin{cases}1 & \text { if } F=0 \text { and } \dot{\lambda}>0 \text { (plastic loading) } \\
0 & \text { otherwise }\end{cases}
\end{array}
$$

and,

$$
\begin{aligned}
b_{k l}^{F} & =\frac{\partial F}{\partial \tau_{i j}} C_{i j k l}^{e} \\
b_{i j}^{Q} & =C_{i j k l}^{e} \frac{\partial Q}{\partial \tau_{k l}} \\
b_{k l}^{F M} & =\frac{\partial F}{\partial \mu_{i j}} M_{i j k l}^{e} \\
b_{i j}^{Q M} & =M_{i j k l}^{e} \frac{\partial Q}{\partial \mu_{k l}}
\end{aligned}
$$


Using Eqs. 3.8 and 3.12, the incremental constitutive equations can be expressed as:

$$
\begin{gathered}
\dot{\tau}_{i j}=\left(C_{i j k l}^{e}-\frac{<1>}{H_{p}} b_{i j}^{Q} b_{k l}^{F}\right) \dot{\gamma_{k l}}-\alpha \dot{T}\left(C_{i j k l}^{e}-\frac{<1>}{H_{p}} b_{i j}^{Q} b_{k l}^{F}\right) \delta_{k l}-\frac{<1>}{H_{p}} b_{i j}^{Q} b_{k l}^{F M} \dot{\kappa}_{k l} \\
\dot{\mu}_{i j}=\left(M_{i j k l}^{e}-\frac{<1>}{H_{p}} b_{i j}^{Q M} b_{k l}^{F M}\right) \dot{\kappa}_{k l}-\frac{<1>}{H_{p}} b_{i j}^{Q M} b_{k l}^{F} \dot{\gamma}_{k l}-\alpha \dot{T} \frac{<1>}{H_{p}} b_{i j}^{Q M} b_{k l}^{F} \delta_{k l}
\end{gathered}
$$

These constitutive relationships can be written in the general form.

$$
\begin{gathered}
\dot{\tau}_{i j}=C_{i j k l}^{e p} \dot{\gamma}_{k l}+D_{i j k l}^{e p} \dot{\kappa}_{k l}+E_{i j k l}^{e p} \dot{T} \delta_{k l} \\
\dot{\mu}_{i j}=M_{i j k l}^{e p} \dot{\kappa}_{k l}+L_{i j k l}^{e p} \dot{\gamma}_{k l}+N_{i j k l}^{e p} \dot{T} \delta_{k l}
\end{gathered}
$$

with,

$$
\begin{array}{cc}
C_{i j k l}^{e p}=C_{i j k l}^{e}-\frac{<1>}{H_{p}} b_{i j}^{Q} b_{k l}^{F} & D_{i j k l}^{e p}=-\frac{<1>}{H_{p}} b_{i j}^{Q} b_{k l}^{F M} \\
E_{i j k l}^{e p}=-\left(C_{i j k l}^{e}-\frac{<1>}{H_{p}} b_{i j}^{Q} b_{k l}^{F}\right) & L_{i j k l}^{e p}=-\frac{<1>}{H_{p}} b_{i j}^{Q M} b_{k l}^{F} \\
M_{i j k l}^{e p}=M_{i j k l}^{e}-\frac{<1>}{H_{p}} b_{i j}^{Q M} b_{k l}^{F M} & N_{i j k l}^{e p}=\frac{<1>}{H_{p}} b_{i j}^{Q M} b_{k l}^{F}
\end{array}
$$

\subsection{Drucker-Prager yield surface}

The classical Drucker-Prager plastic model for cohesionless materials was extended to Cosserat media by Mühlhaus and Vardoulakis (1987) for a 2D continuum. Herein, we follow the same approach to develop a 3D THM model for Cosserat continua.

$$
F=\tau+\mu \sigma \quad \text { and } \quad Q=\tau+\beta \sigma
$$

The generalized stress invariants are defined as:

$$
\begin{aligned}
\sigma & =\frac{\tau_{k k}}{3} \\
\tau & =\sqrt{h_{1} s_{i j} s_{i j}+h_{2} s_{i j} s_{j i}+\frac{1}{R^{2}}\left(h_{3} m_{i j} m_{i j}+h_{4} m_{i j} m_{j i}\right)}
\end{aligned}
$$

Similarly, the generalized plastic deviatoric strain rate is written as:

$$
\dot{\gamma}_{p}=\sqrt{g_{1} \dot{e}_{i j}^{p} \dot{e}_{i j}^{p}+g_{2} \dot{e}_{i j}^{p} \dot{e}_{j i}^{p}+R^{2}\left(g_{3} \dot{k}_{i j}^{p} \dot{k}_{i j}^{p}+g_{4} \dot{k}_{i j}^{p} \dot{k}_{j i}^{p}\right)}
$$


strain and curvature respectively. The evaluation of the parameters $h_{i}$ and $g_{i}$ is discussed in Appendix B based on micro-mechanical considerations. Some values are summarized in Table 1.

\begin{tabular}{|c|c|c|}
\hline & 2D model & 3 D model \\
\hline static model & $\left\{h_{i}\right\}=\{3 / 4,-1 / 4,1,0\}$ & $\left\{h_{i}\right\}=\{2 / 3,-1 / 6,2 / 3,-1 / 6\}$ \\
& $\left\{g_{i}\right\}=\{3 / 2,1 / 2,1,0\}$ & $\left\{g_{i}\right\}=\{8 / 5,2 / 5,8 / 5,2 / 5\}$ \\
\hline Kinematic model & $\left\{h_{i}\right\}=\{3 / 8,1 / 8,1 / 4,0\}$ & $\left\{h_{i}\right\}=\{2 / 5,1 / 10,2 / 5,1 / 10\}$ \\
& $\left\{g_{i}\right\}=\{3,-1,4,0\}$ & $\left\{g_{i}\right\}=\{8 / 3,-2 / 3,8 / 3,-2 / 3\}$ \\
\hline
\end{tabular}

Table 1: Values for the coefficients in the stress and strain deviatoric generalized invariants for a Cosserat continuum from Mühlhaus (1986), Mühlhaus and Vardoulakis (1987), Sulem and Vardoulakis (1990), Unterreiner (1994)

The hardening shear modulus is:

$$
-H_{s}=\frac{\partial F}{\partial \gamma^{p}}=\frac{\partial \mu}{\partial \gamma^{p}} \sigma
$$

and the hardening coefficient is:

$$
h_{s}=\frac{\partial \mu}{\partial \gamma^{p}}
$$

The gradient terms of the yield function and plastic potential are expressed as :

$$
\begin{array}{r}
\frac{\partial F}{\partial \tau_{i j}}=\frac{1}{\tau}\left(h_{1} s_{i j}+h_{2} s_{j i}\right)+\frac{\mu}{3} \delta_{i j} \\
\frac{\partial Q}{\partial \tau_{i j}}=\frac{1}{\tau}\left(h_{1} s_{i j}+h_{2} s_{j i}\right)+\frac{\beta}{3} \delta_{i j} \\
\frac{\partial F}{\partial \mu_{i j}}=\frac{\partial Q}{\partial \mu_{i j}}=\frac{1}{\tau R^{2}}\left(h_{3} \mu_{i j}+h_{4} \mu_{j i}\right)
\end{array}
$$

It is easily shown that $\dot{\lambda}=\dot{\gamma}^{p}$ and $\dot{\varepsilon}_{v}^{p}=\beta \dot{\gamma}^{p}$ by decomposing $\dot{\gamma}_{i j}^{p}$ (obtained from the plastic flow rule) into its deviatoric and volumetric part and then replacing them into the expression for $\dot{\gamma}^{p}$. Moreover, we have

$b_{k l}^{F}=K \mu \delta_{k l}+\frac{s_{k l}}{q}\left(\left(G+G_{c}\right) h_{1}+\left(G-G_{c}\right) h_{2}\right)+\frac{s_{l k}}{q}\left(\left(G+G_{c}\right) h_{2}+\left(G-G_{c}\right) h_{1}\right)$ 


$$
\begin{gathered}
b_{i j}^{Q}=K \beta \delta_{i j}+\frac{s_{i j}}{\tau}\left(\left(G+G_{c}\right) h_{1}+\left(G-G_{c}\right) h_{2}\right)+\frac{s_{j i}}{\tau}\left(\left(G+G_{c}\right) h_{2}+\left(G-G_{c}\right) h_{1}\right) \\
b_{k l}^{F M}=b_{k l}^{Q M}=\frac{1}{\tau R^{2}}\left[m_{k l}\left(\left(M+M_{c}\right) h_{3}+\left(M-M_{c}\right) h_{4}\right)+m_{l k}\left(\left(M-M_{c}\right) h_{3}+\left(M+M_{c}\right) h_{4}\right)\right] \\
\begin{array}{c}
\frac{\partial F}{\partial \sigma_{i j}} C_{i j k l} \frac{\partial Q}{\partial \sigma_{k l}}=K \mu \beta+\frac{s_{k l} s_{k l}}{\tau^{2}}\left(\left(G+G_{c}\right)\left(h_{1}^{2}+h_{2}^{2}\right)+2\left(G-G_{c}\right) h_{1} h_{2}\right) \\
+\frac{s_{k l} s_{l k}}{\tau^{2}}\left(\left(G-G_{c}\right)\left(h_{1}^{2}+h_{2}^{2}\right)+2\left(G+G_{c}\right) h_{1} h_{2}\right) \\
\frac{\partial F}{\partial \mu_{i j}} M_{i j k l} \frac{\partial Q}{\partial \mu_{k l}}=\frac{1}{\tau^{2} R^{4}}\left[m_{k l} m_{k l}\left(\left(M+M_{c}\right)\left(h_{3}^{2}+h_{4}^{2}\right)+2\left(M-M_{c}\right) h_{3} h_{4}\right)\right. \\
\left.+m_{k l} m_{l k}\left(\left(M-M_{c}\right)\left(h_{3}^{2}+h_{4}^{2}\right)+2\left(M+M_{c}\right) h_{3} h_{4}\right)\right]
\end{array}
\end{gathered}
$$

\section{Bifurcation analysis}

Let us consider an evolution problem described by Eqs. 2.4, 2.5, 2.8 and 2.17. This set of equations presents a homogeneous steady state without Cosserat effects if adiabatic, undrained and prescribed total stresses as boundary conditions are applied. Note that these boundary conditions are different from Lachenbruch (1980), Rice et al. (2014) as they consider a layer sheared at a constant strain rate. The steady state is defined by $T=T_{s}, p=p_{s}, \tau_{i j}=\tau_{i j}^{0}$, $\gamma_{i j}=\gamma_{i j}^{0}, \mu_{i j}=0$ and $\kappa_{i j}=0$, where $T_{s}$ and $p_{s}$ are a reference temperature and pressure respectively, $\tau_{i j}^{0}$ and $\gamma_{i j}^{0}$ are the homogeneous stress and deformation tensors that depend on the geometry and boundary conditions. We denote all the fields corresponding to this steady state with a superscript 0 .

We are interested in determining the conditions for which the above homogeneous solutions become unstable in the Lyapunov sense (Lyapunov, 1892, 
Stefanou and Alevizos, 2016). The relevant variables of the problem are expressed as follows:

$$
\begin{array}{r}
T\left(x_{i}, t\right)=T^{0}+T^{*}\left(x_{i}, t\right) \quad p\left(x_{i}, t\right)=p^{0}+p^{*}\left(x_{i}, t\right) \\
\tau_{k l}^{\prime}\left(x_{i}, t\right)=\tau_{k l}^{\prime 0}+\tau_{k l}^{\prime *}\left(x_{i}, t\right) \\
u_{k}\left(x_{i}, t\right)=u_{k}^{0}\left(x_{i}\right)+u_{k}^{*}\left(x_{i}, t\right) \\
\omega_{k}^{c}\left(x_{i}, t\right)=\omega_{k}^{c 0}+\omega_{k}^{c *}\left(x_{i}, t\right)
\end{array}
$$

225

The constitutive equations 3.20 can be linearized around the reference state to obtain the following relationships between the perturbations of stresses, couple stresses, strains and curvatures:

$$
\begin{aligned}
\tau_{k l}^{\prime *} & =C_{k l m n}^{e p} \gamma_{m n}^{*}+D_{k l m n}^{e p} \kappa_{m n}^{*}+E_{k l m n}^{e p} T^{*} \delta_{m n} \\
\mu_{k l}^{*} & =M_{k l m n}^{e p} \kappa_{m n}^{*}+L_{k l m n}^{e p} \gamma_{m n}^{*}+N_{k l m n}^{e p} T^{*} \delta_{m n}
\end{aligned}
$$

Eqs. 2.4, 2.5, 2.8 and 2.17 become then:

$$
\begin{array}{r}
C_{k l m n}^{e p}\left(u_{m, n l}^{*}+e_{m n q} \omega_{q, l}^{c *}\right)+E_{k l m n}^{e p} T_{, l}^{*} \delta_{m n}+D_{k l m n}^{e p} \omega_{m, n l}^{c *}-p_{, l}^{*} \delta_{k l}-\rho \frac{\partial^{2} u_{k}^{*}}{\partial t^{2}}=0 \\
M_{k l m n}^{e p} \omega_{m, n l}^{c *}+L_{k l m n}^{e p}\left(u_{m, n l}^{*}+e_{m n q} \omega_{q, l}^{c *}\right)+N_{k l m n}^{e p} T_{, l}^{*} \delta_{m n} \\
-e_{k l m}\left(C_{l m n q}^{e p}\left(u_{n, q}^{*}+e_{n q r} \omega_{r}^{c *}\right)+E_{l m n q}^{e p} T^{*} \delta_{n q}+D_{l m n q}^{e p} \omega_{n, q}^{c *}\right)-\rho I \frac{\partial^{2} \omega_{i}^{c *}}{\partial t^{2}}=0 \\
(4.4) \\
\mathrm{C}\left(\frac{\partial T^{*}}{\partial t}-c_{t h} T_{, k k}^{*}\right)=\tau_{k l}^{\prime 0}\left(\dot{u}_{k, l}^{*}+e_{k l m} \dot{\omega}_{m, l}^{c *}\right)+\mu_{k l}^{0} \dot{\omega}_{k, l}^{c *} \\
\frac{\partial p^{*}}{\partial t}=c_{h y} p_{, k k}^{*}+\Lambda \frac{\partial T^{*}}{\partial t}-\frac{1}{\beta^{*}} \frac{\partial u_{k, k}^{*}}{\partial t}
\end{array}
$$

To obtain Eq. 4.5, we neglect the perturbations of the elastic deformation and curvature tensors as compared to the plastic ones. 
The system of Eqs. $4.3-4.6$ is a linear system of eight equations with eight unknowns (the perturbed fields). It is convenient to apply a space Fourier transform to study it. The system admits solutions of the form:

$$
X_{k}^{*}\left(x_{l}, t\right)=\bar{X}_{k}(t) \cdot \exp \left(i \xi x_{l} n_{l}\right)
$$

where $\xi$ is the inverse of the wavelength $\lambda=\frac{h}{2 \pi N}(N$ is an integer satisfying the boundary conditions). In the following, the height $h$ will be kept as an open parameter so that all values of $\lambda$ will be explored. $n_{j}$ is a polarization direction, $i^{2}=-1$ and $X_{i}^{*}\left(x_{j}, t\right)$ a vector containing the eight perturbation unknowns as follows:

$$
X_{k}^{*}\left(x_{l}, t\right)=\left[u_{k}^{*}\left(x_{l}, t\right) \quad \omega_{k}^{c *}\left(x_{l}, t\right) \quad T^{*}\left(x_{l}, t\right) \quad p^{*}\left(x_{l}, t\right)\right]^{t}
$$

Inserting Eq. 4.7 in Eqs. 4.3 - 4.6, we obtain a system of ordinary differential equations, which admits solutions of the form:

$$
\bar{X}_{k}(t)=\bar{X}_{k} \cdot \exp (s t)
$$

where $s$ is the rate of growth of the perturbation and $\bar{X}_{k}$ a vector of algebraic quantities. This leads to the following linear system of equations, written in matrix form:

$$
\left[\begin{array}{cccc}
\Gamma_{k m}-\rho s^{2} \delta_{k m} & \Delta_{k m} & E_{k l m n}^{e p} i \xi n_{l} \delta_{m n} & -i \xi n_{k} \\
\Xi_{k m} & \Pi_{k m}-\rho I s^{2} \delta_{k m} & -e_{k l m} E_{l m n q}^{e p} \delta_{n q}+N_{k l m n}^{e p} \delta_{m n} & 0 \\
-\tau_{k l}^{\prime 0} s i \xi n_{l} \delta_{k m} & -\tau_{k l}^{\prime 0} s i e_{k l m}-\mu_{k l}^{0} s i \xi n_{l} \delta_{k m} & \rho C\left(s+c_{t h} \xi^{2}\right) & 0 \\
\frac{1}{\beta^{*}} s i \xi n_{k} & 0 & -\Lambda s & s+c_{h y} \xi^{2}
\end{array}\right]\left[\begin{array}{c}
\bar{u}_{m} \\
\bar{\omega}_{m}^{c} \\
\bar{T} \\
\bar{p}
\end{array}\right]=0
$$

where 


$$
\begin{array}{r}
\Gamma_{k m}=-C_{k l m n}^{e p} \xi^{2} n_{n} n_{l} \\
\Delta_{k m}=C_{k l q n}^{e p} e_{q n m} i \xi n_{l}-D_{k l m n}^{e p} \xi^{2} n_{n} n_{l} \\
\Xi_{k m}=-L_{k l m n}^{e p} \xi^{2} n_{n} n_{l}-e_{k l r} C_{l r m q}^{e p} i \xi n_{q} \\
\Pi_{k m}=-M_{k l m n}^{e p} \xi^{2} n_{n} n_{l}+L_{k l r n}^{e p} e_{r n m} i \xi n_{l}-e_{k l r} C_{l r n q}^{e p} e_{n q m}-e_{k l r} D_{l r m q}^{e p} i \xi n_{q}
\end{array}
$$

least one of the roots has a real part equal to 0 and all the others have a real part strictly negative.

Note that the classical condition of localization for rate-independent plastic materials with a Cauchy continuum is retrieved (Rudnicki and Rice, 1975) (no couplings or Cosserat effect considered), i.e. $\operatorname{det}\left(\Gamma_{k m}\right)=0$, where $\Gamma_{k m}$ is the acoustic tensor. The localization condition for a purely mechanical system in the framework of Cosserat continuum is:

$$
\operatorname{det}\left[\begin{array}{cc}
\Gamma_{k m}-\rho s^{2} \delta_{k m} & \Delta_{k m} \\
\Xi_{k m} & \Pi_{k m}-\rho I s^{2} \delta_{k m}
\end{array}\right]=0
$$

The singularity of the above tensor is similar to the condition found in Iordache and William (1998), Steinmann and Willam (1991) for the onset of localization $250(s=0)$. In these papers, the authors derive the localization condition from the kinematic and static compatibility conditions across the shear band as done classically for strain localization analysis (Vardoulakis and Sulem, 1995). 


\section{Application to rapid shearing in fault zones}

Field observations of faults show that shear deformation is extremely localmaterial due to excessive shearing (Engelder, 1974, Myers and Aydin, 2004). Outcrops indicate that an even thinner zone of ultracataclastic material is often identified inside the fault core called the principal slip zone (PSZ) (Rice, 2006). The thickness of the PSZ appears to be a key parameter for understanding behavior (Kanamori and Brodsky, 2004) as it is related to the triggering and evolution of various multi-physical couplings and energy dissipation during seismic slip. From the mechanics point of view, the deformations are localized in a shear band whose thickness can be measured from field observations and laboratory tests.

\subsection{Fault core model under THM couplings}

A simple configuration of a fault core is represented in Fig. 2 as a homogeneous infinite layer of fluid saturated granular material with a thickness $h$. The material inside this layer is modeled as a Cosserat continuum in order to take into account its granular microstructure.

Prior to localization, the state of stress, strain, pore pressure and temperature is assumed to be homogeneous in the layer. As a condition of zero couple stress is applied at the boundaries, the couple stresses are identically zero in the medium. Thus, the medium behaves like a Cauchy continuum.

$$
\tau_{12}=\tau_{21} \quad \mu_{i j}=0
$$

Moreover, the different fields depend only on the component $x_{2}$ due to the invariance in the $x_{1}$ and $x_{3}$ directions. Eqs. 3.31 to 3.35 can be simplified at 


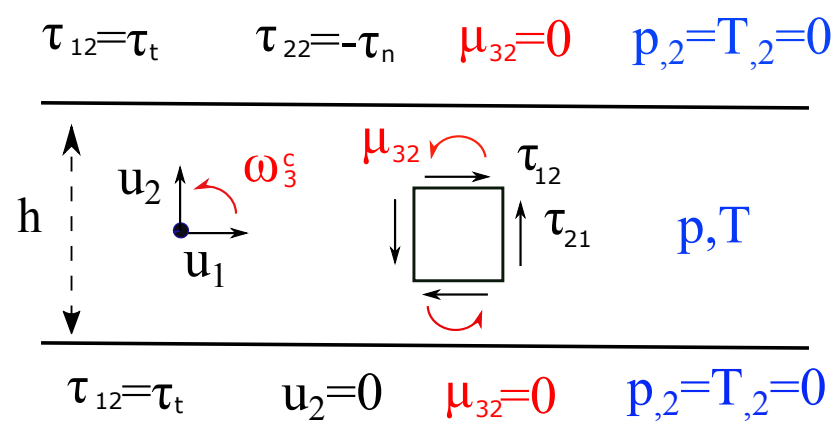

Figure 2: Boundary conditions for the infinite sheared layer modeling a saturated gouge

the homogeneous state:

$$
\begin{gathered}
\frac{\partial F}{\partial \sigma_{i j}} C_{i j k l} \frac{\partial Q}{\partial \sigma_{k l}}=\mu \beta K+G \\
\frac{\partial F}{\partial m_{i j}} M_{i j k l} \frac{\partial Q}{\partial m_{k l}}=0
\end{gathered}
$$

275 SO,

$$
H_{p}=\mu \beta K+G+H_{s}
$$

Moreover,

$$
\begin{gathered}
b_{i j}^{Q M}=b_{k l}^{F M}=0 \\
b_{i j}^{Q} b_{k l}^{F}=K^{2} \mu \beta \delta_{i j} \delta_{k l}+\frac{K \mu G}{\tau} s_{i j} \delta_{k l}+\frac{K \beta G}{\tau} \delta_{i j} s_{k l}+\frac{G^{2}}{\tau^{2}} s_{i j} s_{k l}
\end{gathered}
$$

We introduce the following dimensionless quantities:

$$
\begin{aligned}
& x=\frac{x_{1}}{R} ; \quad z=\frac{x_{2}}{R} ; \quad \overline{\bar{u}}_{i}=\frac{\bar{u}_{i}}{R} ; \quad \overline{\bar{p}}=\frac{\bar{p}}{\tau_{n}} ; \\
& \overline{\bar{\tau}}_{i j}=\frac{\bar{\tau}_{i j}}{\tau_{n}} ; \quad \bar{t}=\frac{c_{t h}}{R^{2}} t ; \quad \overline{\bar{T}}=\frac{\Lambda}{\tau_{n}} \bar{T} ; \quad \bar{\xi}=\xi R
\end{aligned}
$$


Considering Eq. 5.7, the dimensionless form of the matrix in 4.10 is:

$$
\left[\begin{array}{cccc}
\frac{1}{\tau_{n}} \Gamma_{k m}-I s^{2} & \tilde{\Delta}_{k m} & \frac{1}{\Lambda} E_{k l m n}^{e p} i \bar{\xi} n_{l} \delta_{m n} & -i \bar{\xi} n_{i} \\
\tilde{\Xi}_{k m} & \tilde{\Pi}_{k m}-\frac{I}{2} s^{2} & \frac{1}{\Lambda R}\left(-R e_{k l m} E_{l m n q}^{e p} \delta_{n q}+N_{k l m n}^{e p} \delta_{m n}\right) & 0 \\
-\alpha_{k l} s i \bar{\xi} n_{l} \delta_{k m} & -\frac{1}{R} \alpha_{k l} s i e_{k l m}-\zeta_{k l} s i \bar{\xi} n_{l} \delta_{k m} & s+\bar{\xi}^{2} & 0 \\
\frac{1}{\beta^{*} \tau_{n}} s i \bar{\xi} n_{k} & 0 & -s & s+\frac{1}{L e} \bar{\xi}^{2}
\end{array}\right]
$$

where,

$$
\begin{array}{r}
\tilde{\Delta}_{i k}=\frac{1}{\tau_{n}}\left(C_{i j q l}^{e p} e_{q l k} i \xi n_{j}-\frac{1}{R} D_{i j k l}^{e p} \xi^{2} n_{l} n_{j}\right) \\
\tilde{\Xi}_{i k}=\frac{1}{\tau_{n}}\left(-\frac{1}{R} L_{i j k l}^{e p} \xi^{2} n_{l} n_{j}-e_{i j q} C_{j q k m}^{e p} i \xi n_{m}\right) \\
\tilde{\Pi}_{i k}=\frac{1}{\tau_{n}}\left(-\frac{1}{R^{2}} M_{i j k l}^{e p} \xi^{2} n_{l} n_{j}+\frac{1}{R} L_{i j q l}^{e p} e_{q l k} i \xi n_{j}-e_{i j q} C_{j q l m}^{e p} e_{l m k}-\frac{1}{R} e_{i j q} D_{j q k m}^{e p} i \xi n_{m}\right) \\
\alpha_{i j}=\frac{\tau_{i j}^{\prime 0} \Lambda}{\rho C \tau_{n}} ; \quad \zeta_{i j}=\frac{\mu_{i j}^{0} \Lambda}{\rho C \tau_{n} R} ; \quad L e=\frac{c_{t h}}{c_{h y}}
\end{array}
$$

Note that for an infinite layer, as we assume invariance in $x_{1}$ and $x_{3}$ direction, ure a positive real part. For $H_{s}<H_{c r}$, some real roots are positive (Figure 3). 


\begin{tabular}{|c|c|c||c|c|c|}
\hline parameters & values & units & parameters & values & units \\
\hline$K$ & $16.67 \times 10^{3}$ & $\mathrm{MPa}$ & $\mu$ & 0.5 & \\
$G$ & $10 \times 10^{3}$ & $\mathrm{MPa}$ & $\beta$ & 0 & \\
$G c$ & $5 \times 10^{3}$ & $\mathrm{MPa}$ & $\lambda^{*}$ & $7.4 \times 10^{-5}$ & $/{ }^{\circ} \mathrm{C}$ \\
$R$ & 0.01 & $\mathrm{~mm}$ & $\rho C$ & 2.8 & $\mathrm{MPa} /{ }^{\circ} \mathrm{C}$ \\
$\rho$ & 2500 & $\mathrm{~kg} / \mathrm{m}^{3}$ & $c_{t h}$ & 1 & $\mathrm{~mm}^{2} / \mathrm{s}$ \\
$\tau_{n}$ & 200 & $\mathrm{MPa}$ & $c_{h y}$ & 12 & $\mathrm{~mm}^{2} / \mathrm{s}$ \\
$\tau^{\prime 0}$ & 67 & $\mathrm{MPa}$ & $\alpha_{s}$ & $2.5 \times 10^{-5}$ & $/{ }^{\circ} \mathrm{C}$ \\
$\beta^{*}$ & $8.2 \times 10^{-5}$ & $\mathrm{MPa}^{-1}$ & $n$ & 0.04 & \\
\hline
\end{tabular}

Table 2: Numerical values for the parameters of a deep rock gouge from Sulem et al. (2011), Rice (2006)

In Figures 3 and 4, the dashed lines represent the real parts of the roots that have a nonzero imaginary part. For these values the system diverges by oscillating, it corresponds to a so-called "flutter instability". As mentioned in Benallal and Comi (2003), our system is not differentiable due to the KuhnTucker condition (Eq. 4.2), therefore to develop the above equations, we have assumed monotonous loading conditions to stay in the plastic regime. Thus, one cannot assess that the flutter type instabilities obtained in this analysis are meaningful. Moreover, in Simões and Martins (2005), the authors performed linear stability and finite element analyses for a non-associative elastic-plastic layer and observed that the complex eigenvalues with a positive real part, do not always correspond to an unstable behavior in the numerical results.

The value for the critical hardening modulus given in Sulem et al. (2011) is retrieved. The results are very sensitive to the ratio $\lambda^{*} / \beta^{*}$ because shear heating destabilizes the system and instability can occur even in the hardening regime. When plastic shear deformations occur, the energy is dissipated by heat and the temperature in the gouge increases. The presence of the thermal pressurization term $\frac{\lambda^{*}}{\beta^{*}} \frac{\partial \mathrm{T}}{\partial t}$ in the mass balance equation, entails an increase of the pore pressure and thus a decrease of the effective normal stresses if the total 


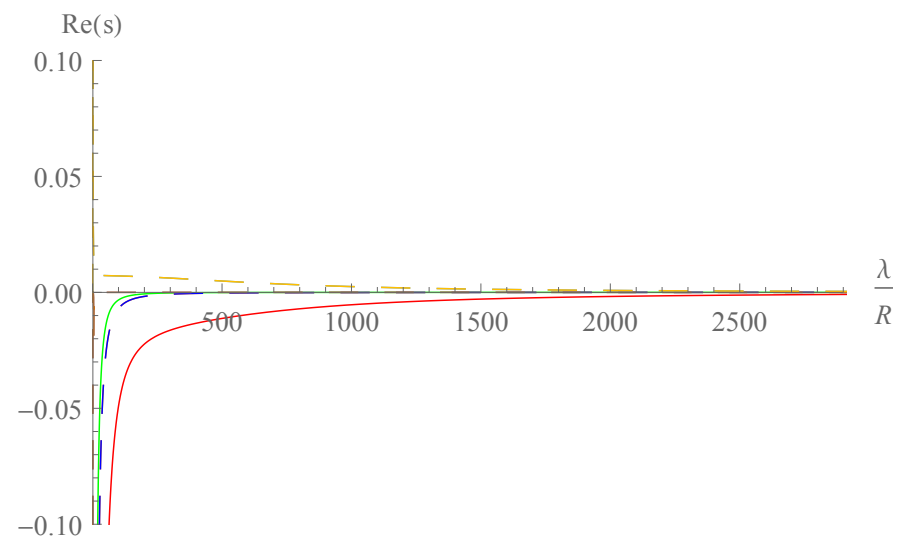

Figure 3: Real parts of the growth coefficient as a function of the wavelength of the perturbation for $H_{s}=2.1 \mathrm{MPa}>H_{c r}$. The different colors represent different roots. The dashed lines represent the real part of the roots with non zero imaginary parts whereas the continuous lines correspond to real roots.

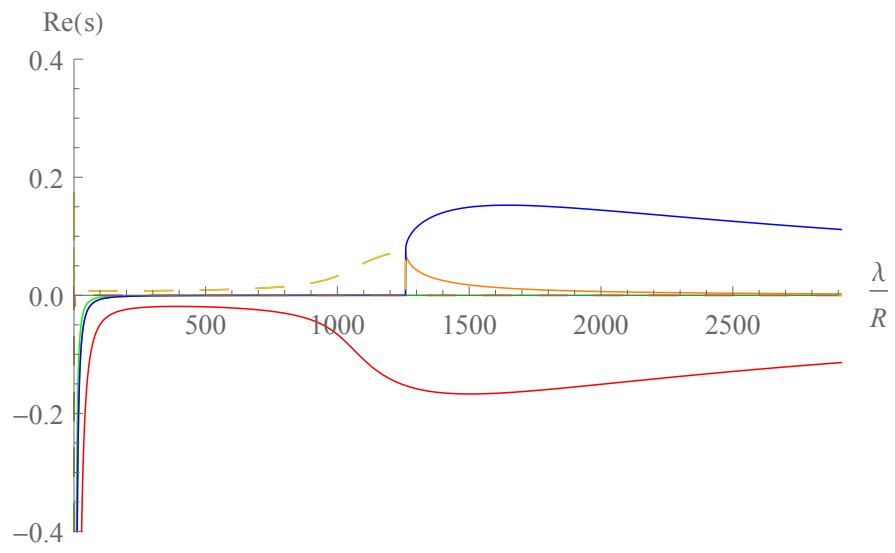

Figure 4: Real parts of the growth coefficient as a function of the wavelength of the perturbation for $H_{s}=1.9 \mathrm{MPa}<H_{c r}$. The different colors represent different roots. The dashed lines represent the real part of the roots with non zero imaginary parts whereas the continuous lines correspond to real roots. 
stress is kept constant. This decrease of the normal stress induces a decrease of the fault strength and destabilizes the system.

For a given hardening modulus below the critical value $H_{s}<H_{c r}$, we can see in Figure 4 that the real roots of the polynomial equation present a maximum. This finite maximal growth coefficient of the instability is obtained thanks to the introduction of the inertia terms (Sulem et al., 2011). This maximum corresponds to the instability mode with fastest growth (see Eq. 4.9). If we consider that the width of the localized zone corresponds to the wavelength of fastest growth, we can plot in Fig. 5 the evolution of the selected wavelength $\lambda_{\max }$ (normalized by the Cosserat material length $\mathrm{R}$ ) as a function of the hardening modulus. It should be mentioned that as argued by Sulem et al. (2011), inertia and micro-inertia terms play a minor role on the onset of the instability and on the selected wavelength. This is also corroborated by (Rice, 2006) and (Rice et al., 2014) who state that inertia terms are negligible over length scales over 325 which thermal and fluid diffusion is important. However, the quasi static approximation reaches its limit because it leads to an infinite rate for the evolution of the perturbation (unbounded growth coefficient in LSA analysis).

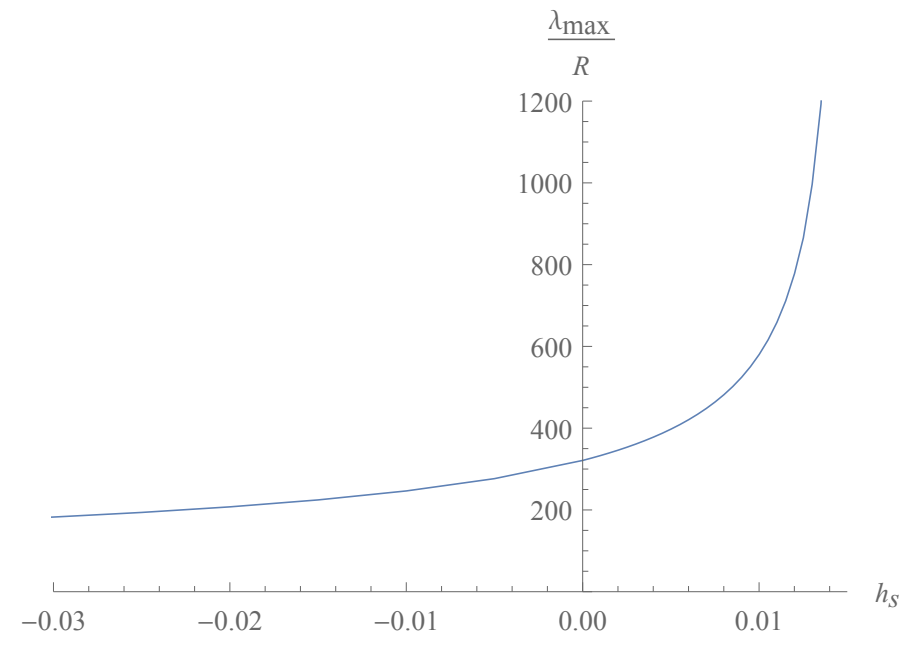

Figure 5: wavelength of the perturbation with fastest growth $\lambda_{\max }$ as a function of the hardening coefficient $h_{s}$ 
The selected wavelength tends to infinity for $H_{s} \rightarrow H_{c r}^{-}$and decreases with decreasing hardening modulus. This general trend is similar to (Mühlhaus and Vardoulakis, 1987). The authors observed that for dry granular materials the shear band thickness is infinite at the bifurcation state and then decreases in the post-bifurcation regime as the strain localization evolves.

When a layer of granular material is sheared, experiments show that the shear stress evolves towards a residual value and, thus, the hardening modulus tends to 0 after sufficient slip (see Fig. 12 and Chambon et al. (2006)). In this example, the width of localization for $H_{s} \rightarrow 0$ is $320 \times R=3.2 \mathrm{~mm}$. Indeed, Rice (2006) stated that most of the models that calculate the width of localization for dry granular materials predict a thickness between 7 to 30 times $D_{50}$ (the mean particle diameter)(Oda and Kazama, 1998, Muir Wood, 2002, Tordesillas

340 et al., 2004) and this is much smaller that what is observed here. However, it should be noted that most of these analyses were performed considering sand and calibrated on data from triaxial experiments performed at much lower confining pressure than the ones acting at a depth of a few kilometers.

\subsection{Effect of friction and dilatancy on localization}

The yield surface for a Drucker-Prager model is defined with two parameters: the friction and the dilatancy coefficients. In this section, we look at their influence on the triggering of localization. The critical hardening parameter, under which the homogeneous state of deformation is unstable for some wavelengths of the perturbation, is plotted as a function of $\beta$ and $\mu$.

In granular materials, inelastic deformations can induce volume changes. In low porosity rocks dilatancy can be the result from rearrangement of close packed particles due to shearing or from uplift sliding over asperity contacts. But the opposite effect, compaction, can also be observed as a result of pore collapse or grain crushing (Rudnicki, 2000). To illustrate the influence of these effects on the stability of our system, we plot on Fig. 6 the value of the bifurcation parameter $H_{c r}$ as a function of the dilatancy coefficient $\beta$ ranging from -0.004 to 0.004 for Mechanical, Hydro-Mechanical and Thermo-Hydro-Mechanical couplings. 
The mechanical system presents a linear evolution of the critical hardening modulus with $\beta$. For a dilatant material $(\beta>0)$, bifurcation occurs in the softening regime, whereas for a compacting material $(\beta<0)$, bifurcation occurs in the hardening regime for a value much greater than the one obtained for $\beta=0$.

For HM couplings, the evolution is bilinear and the two lines intersect at $\beta=0$. The bifurcation is the same as for the mechanical system in the dilatant regime, whereas it is obtained for higher values of $H_{s}$ in the contractant regime. The appearance of this second line in the contractant regime is due to the undrained behavior that becomes unstable before the underlying drained one (Rice, 1975). Indeed, the dilatancy coefficient affects the evolution of pore pressure. If the material is compacting the pore volume decrease induces pore fluid pressurization, which triggers instability.

The introduction of thermal pressurization in the THM model has the effect of shifting the line in the contractant regime to even higher values of $H_{c r}$, but the slope remains the same. Thus, the undrained behavior is affected by thermal pressurization but the drained behavior is not.

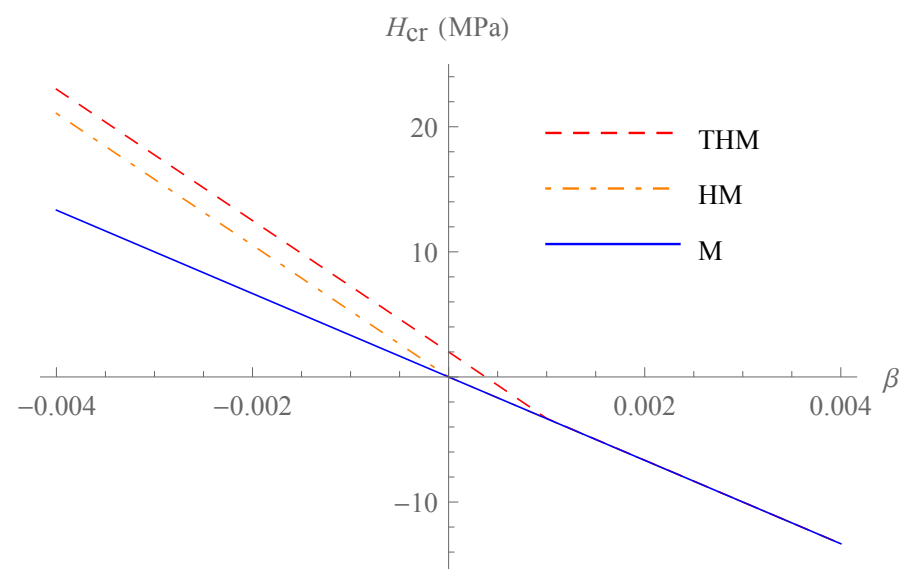

Figure 6: Critical hardening modulus at bifurcation $H_{c r}$ plotted as a function of the dilatancy coefficient $\beta$ considering Mechanical (M), Hydro-Mechanical (HM) and Thermo-HydroMechanical (THM) couplings $(\mu=0.5)$. 

parameter. In Fig. 7, we plot the value of the critical hardening parameter against the initial friction coefficient ranging from 0.3 to 0.8 (typical values obtained experimentally for a gouge (Scott et al., 1994, Scuderi et al., 2013)). For a higher value of $\mu$, the shear stress applied to the sheared layer is greater and thus the mechanical energy dissipated is greater which makes the system more unstable.

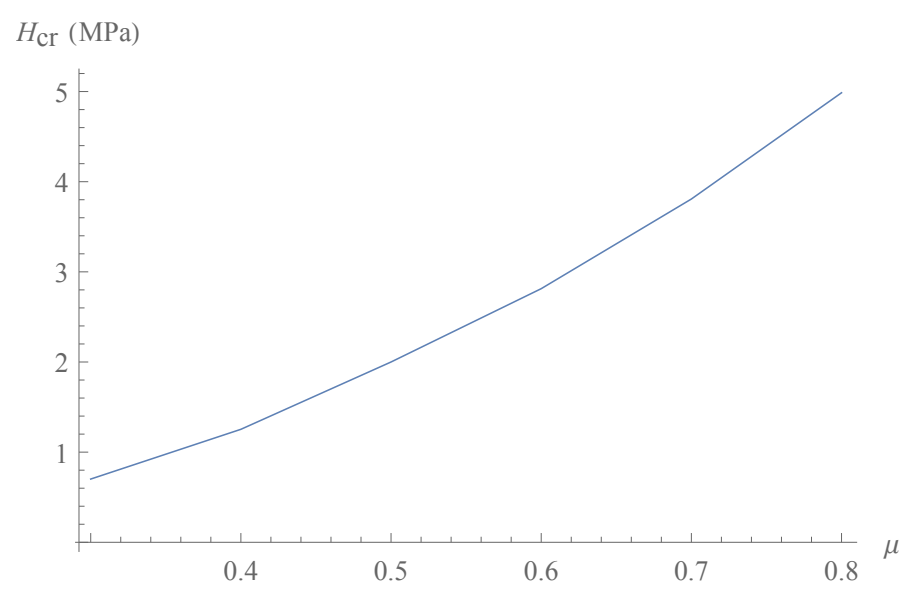

Figure 7: Bifurcation parameter $H_{c r}$ for different values of friction coefficient considering THM couplings $(\beta=0)$.

\subsection{Sensitivity analysis to hydraulic parameters and normal stress}

The hydraulic parameters of the problem are not well constrained, they depend on the material, the pore pressure, the temperature, the porosity, etc... of the system, we plot the evolution of the wavelength selection $\lambda_{\max }$ with the hardening coefficient for different values of them.

Several studies were conducted in order to investigate the value of permeability and diffusivity inside the fault core (Wibberley and Shimamoto, 2003, Sulem et al., 2004, Rafini, 2008). They show that the gouge has a much lower permeability than the surrounding fractured rock mass. It can be three orders 
of magnitude less $\left(10^{-19} \mathrm{~m}^{2}\right.$ for the gouge compared to $10^{-16} \mathrm{~m}^{2}$ for surrounding rock). In Figure 8, the selected wavelength is plotted as a function of the hardening modulus for three values of $\chi$. For a very low permeability $\chi=10^{-21} \mathrm{~m}^{2}$ the results are similar to $\chi=10^{-19} \mathrm{~m}^{2}$. For $\chi=10^{-17} \mathrm{~m}^{2}$, the bifurcation parameter is the same but the significant effect is on the wavelength selection. For $H_{s} \rightarrow 0, \lambda_{\max } / R$ tends towards 373 for $\chi=10^{-17} \mathrm{~m}^{2}$.

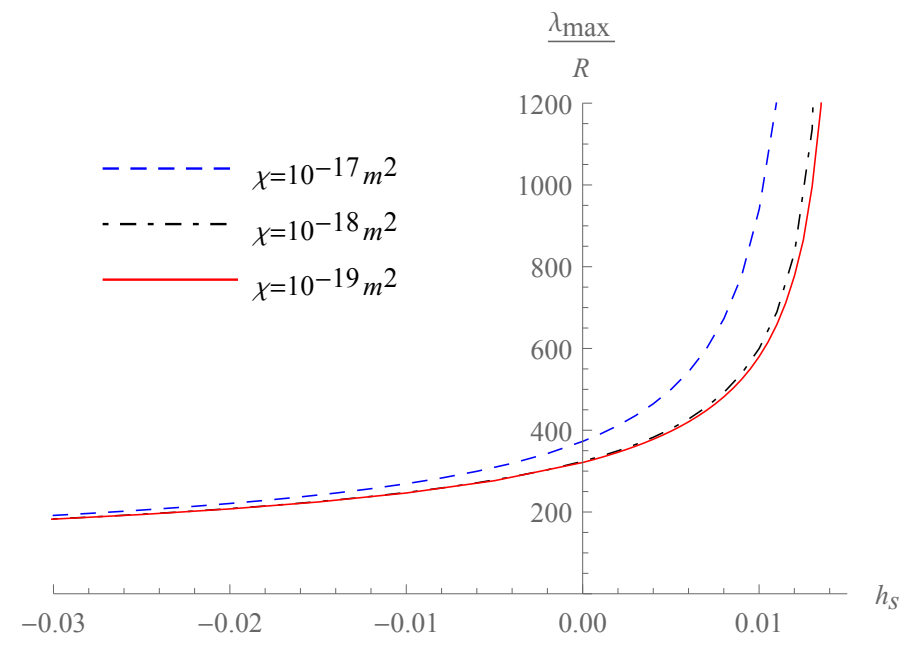

Figure 8: wavelength selection $\lambda_{\max }$ as a function of the hardening coefficient $h_{s}$ for different values of the permeability $\chi$.

Several parameters of the model also depend on the porosity (e.g. the permeability, the thermal pressurization term, the mixture compressibility...). In addition, they evolve during the shear process because of deformations and possible thermally activated chemical reactions (Sulem and Famin, 2009). To take into account the effect of porosity on the permeability, we use a cubic KozenyCarman permeability law.

$$
\chi=\chi_{0}\left(\frac{1-n_{0}}{1-n}\right)^{2}\left(\frac{n}{n_{0}}\right)^{3}
$$

where $\chi_{0}$ and $n_{0}$ are the reference permeability and porosity respectively and their values are the ones considered in Table 2. The other parameters of the model modified by the change of porosity in this study are the ratio $\lambda^{*} / \beta^{*}$ and 
the mixture compressibility. The effect of porosity on the wavelength selection is shown in Figure 9. It has a major effect on the bifurcation parameter and on the shear band width. The greater the porosity, the more unstable the system is. Indeed, when the porosity increases the pore pressure increase due to thermal pressurization is more pronounced and further destabilizes the system.

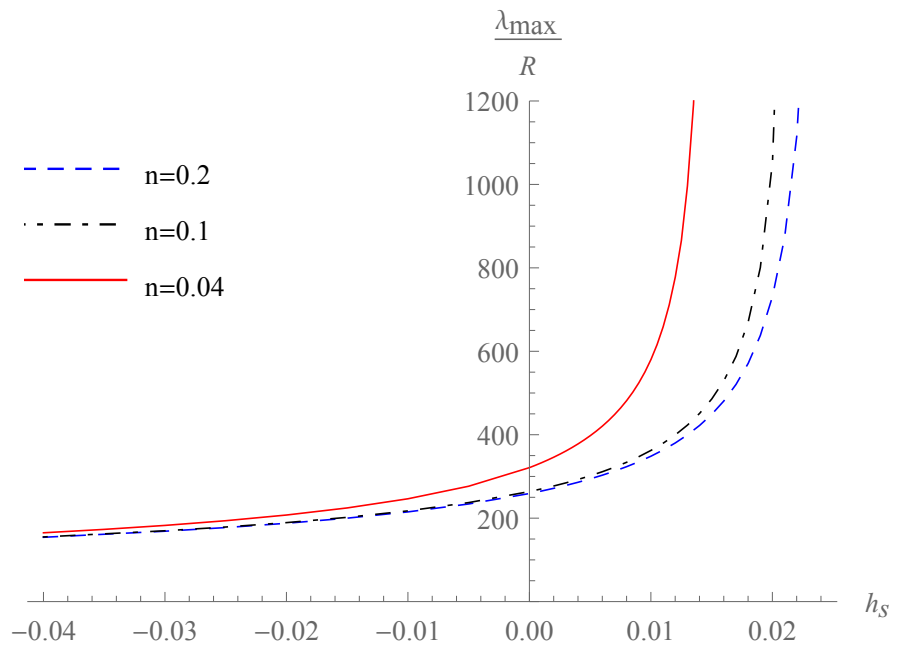

Figure 9: wavelength selection $\lambda_{\max }$ as a function of the hardening coefficient $h_{s}$ for different values of the porosity $n$.

However, the parameter that has the biggest impact on the stability is the ratio $\lambda^{*} / \beta^{*}$ as shown in Figure 10. Considering the different values of the thermal expansion coefficient and of the compressibility for the fluid and the solid given in Rice (2006), this parameter varies between 0.59 and $1.62 \mathrm{MPa} /{ }^{\circ} \mathrm{C}$. We can see that for a value of $0.59 \mathrm{MPa} /{ }^{\circ} \mathrm{C}$, the bifurcation is obtained for a value of the hardening coefficient close to zero $\left(h_{\text {crit }}=0.002\right)$. However, for $\lambda^{*} / \beta^{*}=1.62 \mathrm{MPa} /{ }^{\circ} \mathrm{C}$, the critical value of the hardening coefficient lies clearly in the hardening regime $\left(h_{\text {crit }}=0.045\right)$.

Another effect studied here is the change of the normal stress applied to the sheared layer and its effect on the wavelength selection. We have taken values of 100 and $300 \mathrm{MPa}$ corresponding to 3.5 and 10 kilometers depth in the crust respectively. The highest value corresponds to a typical depth of transition 


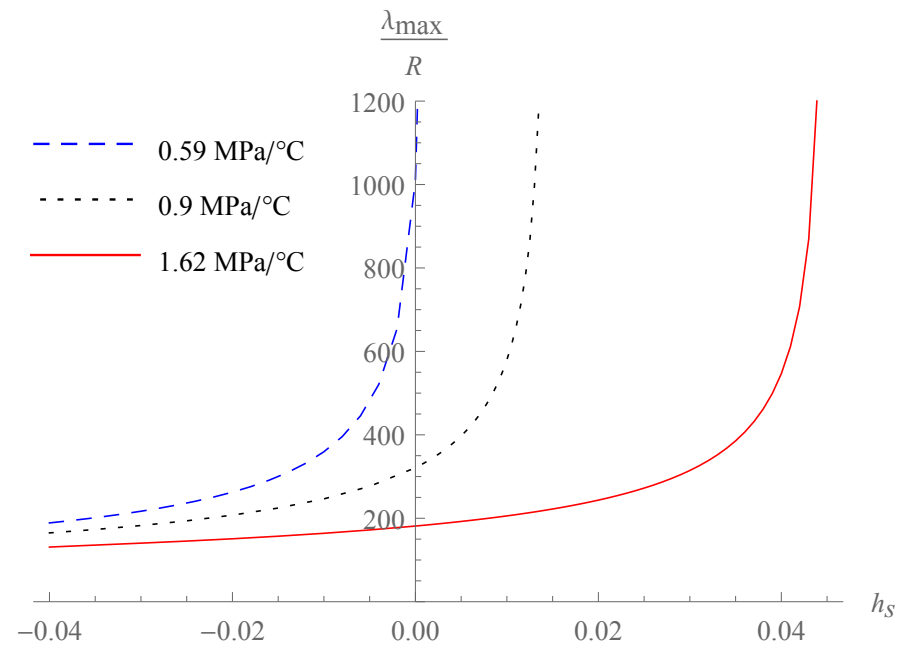

Figure 10: wavelength selection $\lambda_{\max }$ as a function of the hardening coefficient $h_{s}$ for different values of the ratio $\lambda^{*} / \beta^{*}$ that governs the thermal pressurization effect.

between brittle and ductile behavior in the Earth crust. As for the permeability, the bifurcation state is not strongly affected whereas the wavelength selection ${ }_{420}$ is. The greater the normal stress is, the smaller the selected wavelength is and thus the thinner the shear band is. 


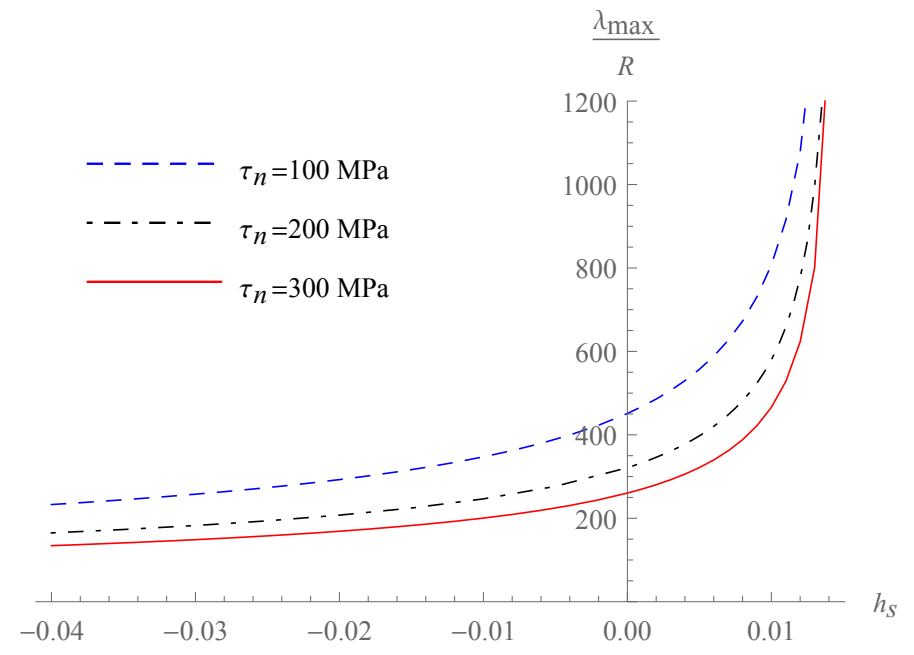

Figure 11: wavelength selection as a function of the hardening coefficient $h_{s}$ for different values of normal stress $\tau_{n}$

\subsection{Evolution of the shear band thickness with the hardening state}

In the previous section, we have studied how the selected wavelength evolves with the hardening modulus for various values of the material parameters. We recall that the above linear stability analysis (LSA) is performed from a reference state which is assumed homogeneous in the system. In the following, we consider a typical stress-strain curve for a fault gouge material under shear and explore the evolution of shear band thickness in due course of the shearing process. This is done by evaluating the selected wavelength from the LSA assuming homogeneous deformation at each state which is not strictly speaking the case because of progressive strain localization. However, it can give an interesting insight which will be confirmed in the companion paper (Rattez et al., 2017) by performing a fully coupled numerical analysis of the post-localization regime.

Ikari et al. (2009) performed double direct shear experiments on a series of saturated fault gouges containing Montmorillonite, Illite, Chlorite and Quartz at effective normal stresses from 12-59 $\mathrm{MPa}$ and at subseismic velocities (1 to $300 \mu \mathrm{m} / \mathrm{s}$ ). In Fig. 12, results for a Montmorillonite-Quartz mixture are presented. From the curve $\tau$ (the tangential stress applied) versus $\gamma$ (the total 
shear deformation), the plastic hardening modulus is related to tangent modulus to the plastic hardening modulus by $h_{s}=\frac{H_{s}}{\tau_{n}}$.

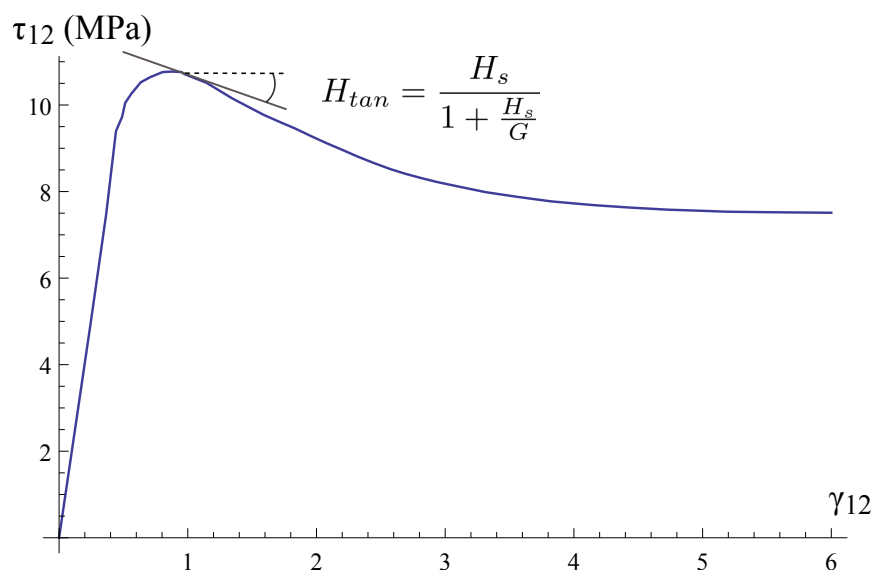

Figure 12: Stress-strain curve for a clay-rich gouge from Ikari et al. (2009).

In Fig. 13, the evolution of the hardening coefficient with the shear deformations obtained from Fig. 12 is shown. It is positive until the maximum shear stress is reached and then negative. Its evolution presents a minimum value of -0.055 , which corresponds to the minimum value of the shear band thickness and then it increases towards zero.

From the evolution of the plastic hardening coefficient in Fig. 13, we can calculate the evolution of the selected wavelength as shown in Fig. 14 for the material parameters considered in Table 2. In Fig.14, we observe that the evolution of the selected wavelength follows the evolution of the plastic hardening coefficient $h_{s}$ : Consequently, the shear band thickness first decreases to a minimum value of $1.5 \mathrm{~mm}$ and then increases to reach a residual value of $3.2 \mathrm{~mm}$. 


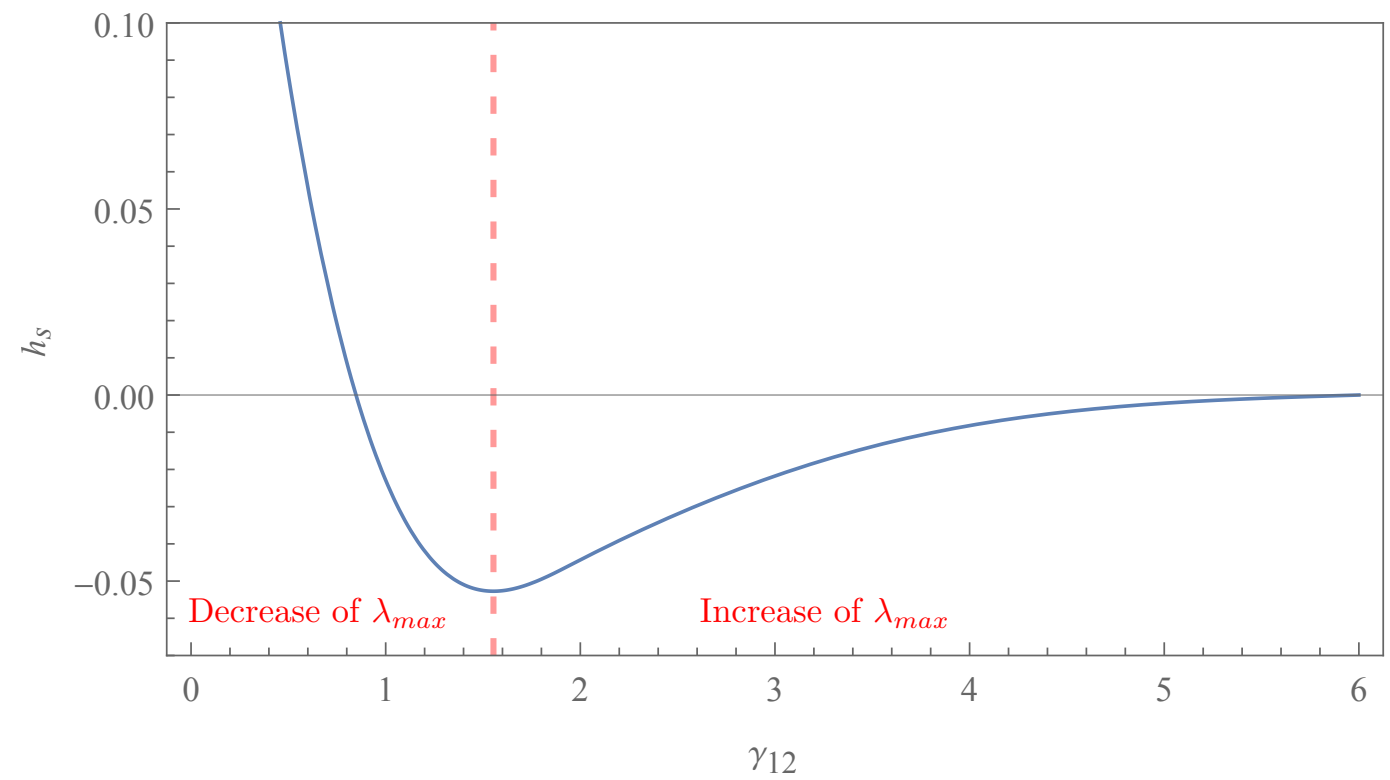

Figure 13: Evolution of the plastic hardening coefficient for the stress-strain response given in Fig. 12.

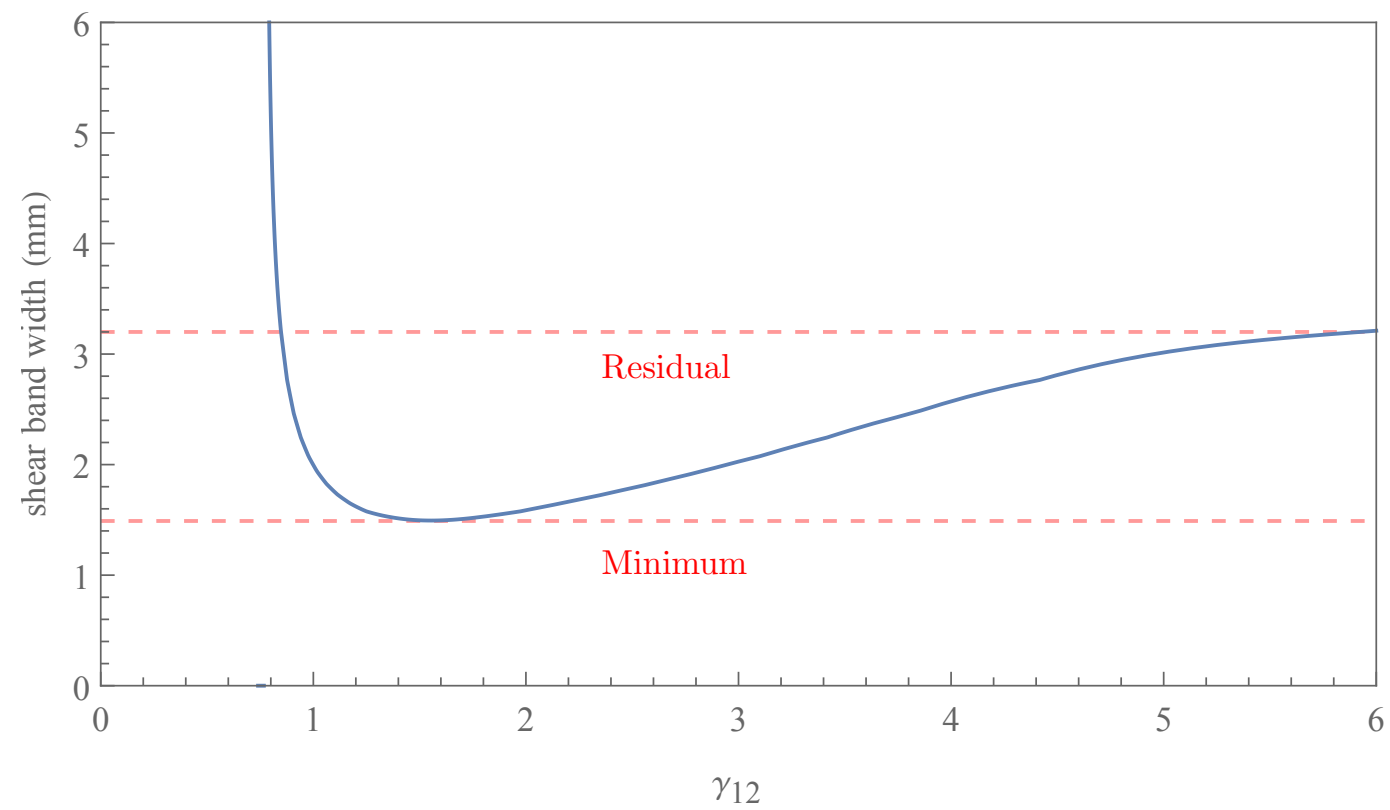

Figure 14: Evolution of the shear band width along with deformations considering the evolution of the hardening coefficient depicted in Fig. 13. 


\subsection{Effect of microstructure evolution}

At high mean stresses, like for faults at great depth, grain crushing is ob-

455 Brantut et al., 2008, Marone and Scholz, 1989). Structural fabrics occurring within fault zones are commonly characterized by distinct grain size and shape distributions that are generally interpreted as strain localization indicators.

Sammis et al. (1987) measured the particle distribution of intact gouge samples, retrieved from the Lopez Fault in the San Gabriel Mountains of Southern California and observed a fractal dimension of approximately 2.6. On the basis of the observations, they proposed the so-called comminution model, for the mechanical processes that generate fault gouges. Several experiments were conducted on natural and simulated fault gouges supporting Sammis' theory (Steacy and Sammis, 1991, An and Sammis, 1994) with some exceptions (Marone and Scholz, 1989, Storti et al., 2003).

The introduction of a characteristic length in the Cosserat continuum enables us to take into account the microstructure evolution of the medium (Bauer, 2016). In our model this characteristic length is related to the mean grain 470 diameter (see Appendix B). Thus, to model a grain size evolution, we assume an exponential decrease with increasing total shear strain $\gamma_{12}$ (Montési and Hirth, 2003).

$$
D\left(\gamma_{12}\right)=\left(D_{0}-D_{f i n}\right) e^{-\frac{\gamma_{12}}{\gamma_{c}}}+D_{f i n}
$$

where $D_{0}$ is the initial grain diameter, $D_{\text {fin }}$ is the final grain diameter and $\gamma_{c}$ is a characteristic deformation that accounts for the rate of evolution (here taken 475 as 1). In Gu and fong Wong (1994), the authors conducted saw-cut experiments on simulated quartz gouges. The particle size distributions of gouge samples before and after frictional sliding were characterized using a laser diffraction particle size analyzer. They obtained a decrease of $30 \%$ of the mean grain diameter after a total shear strain $\gamma_{12}$ of 6 . To see the effect of a more intense 
grain crushing, we study also the case of a decrease of $50 \%$ of the mean grain diameter.

In Fig. 15, we have taken into account the evolution of both the grain size and the hardening modulus with the total shear strain. The decrease of shear band width is almost proportional to the decrease of grain size: for a decrease of $50 \%$ of $D$ we have a decrease of $50 \%$ of the shear band width.

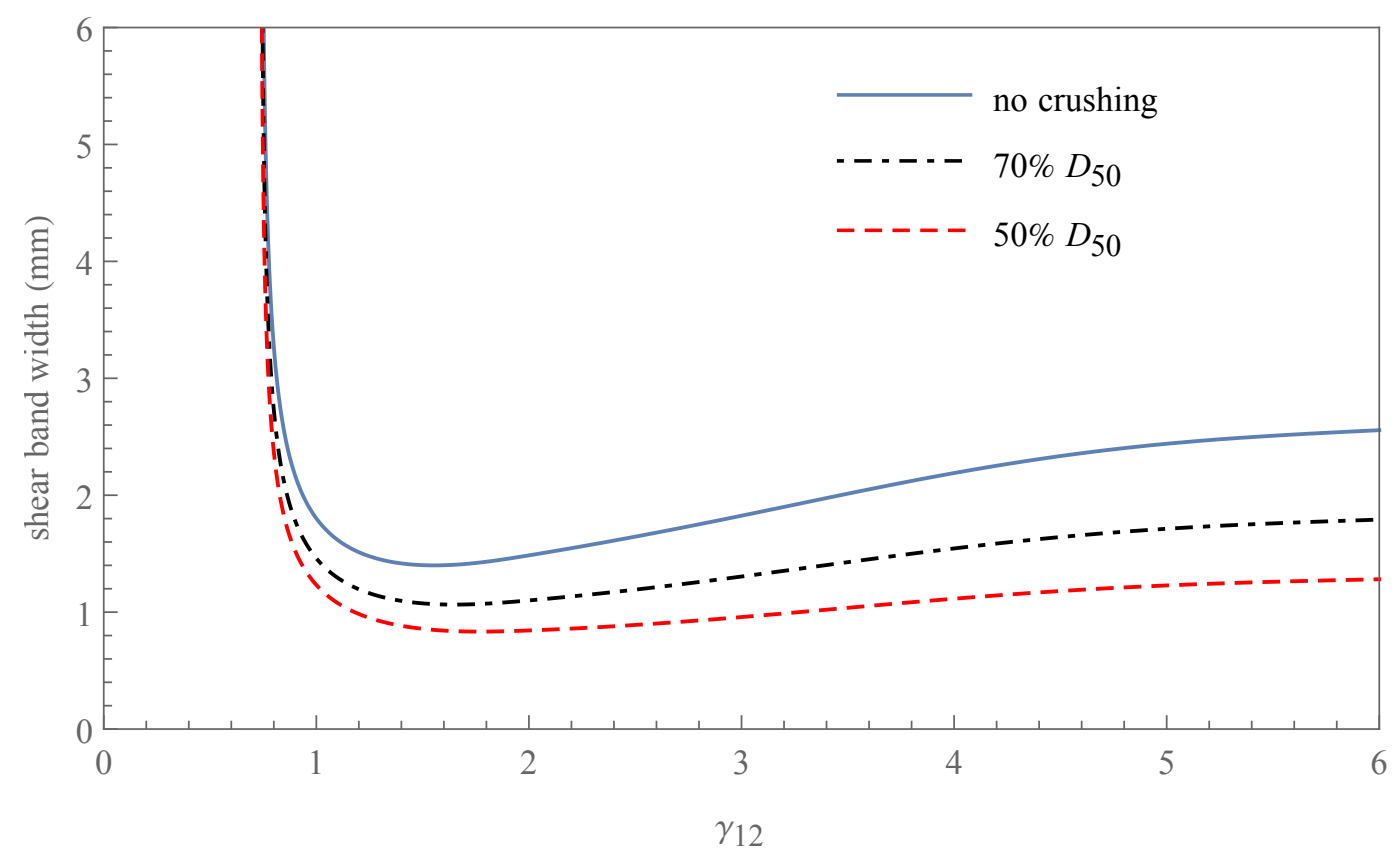

Figure 15: Evolution of the shear band thickness along with deformations considering the evolution of the hardening coefficient as plotted in Fig. 13 and a final mean grain diameter that is $70 \%$ and $50 \%$ of the initial one.

\section{Conclusions}

Seismic slip takes place in a narrow, thin zone (PSZ), whose thickness plays a fundamental role regarding the behavior of ultracataclastic fault cores. In this paper we investigate the effects of the grain size of the fault gouge on the evolution of the thickness of the PSZ. The various Therm-Hydro-Mechanical 
couplings that are ubiquitous during pre- and co-seismic slip of faults are also considered.

Our analysis is based on a three-dimensional Thermo-Hydro-Mechanical model for Cosserat continua. The latter allows to take into account in a direct 
becomes. This intrinsic, physical length scale is introduced by the material parameters of the Cosserat continuum, which can be experimentally determined (see Appendix B). Two additional length scales are intrinsic to the presented model. These lengths emerge from the thermal and hydraulic diffusivities that control also the shear band thickness. In particular the higher these diffusivites are the larger the localization zone is. Consequently, the permeability, which is directly related to the hydraulic diffusivity, influences the size of the shear band. A more permeable medium leads to a broader localization zone. Nevertheless, this effect is small in the range of typical gouge permeabilities.

The role of porosity is twofold regarding localization. Higher porosity commonly corresponds to higher permeability (e.g. Kozeny-Carman law). It also leads to a lower thermal pressurization coefficient. This latter has a more significant influence on the shear band thickness. Higher permeability and lower thermal pressurization coefficient both result in a broader thickness of the localized zone. On the other hand, higher porosity results in a higher mixture compressibility, which on the contrary leads to thinner localized zone. Of course the dominance of one or another of these competing mechanisms depends on the fault material. However, for the material parameters used here, a more porous material presents a narrower localization zone, because the effect of porosity on mixture compressibility is dominant. Nevertheless, the thermal pressurization is not only affected by the porosity but also by other material parameters and the analysis has shown that this parameter has a very significant influence on the localized zone thickness.

The shear band thickness also depends on the confining stress. Although the condition for localization is independent of the confining stress if we assume a linear yield criterion, it affects the shear band thickness. More specifically, large confining stress (e.g. deeper faults) lead to narrower PSZ.

Finally, the evolution of the friction coefficient during shearing has an impact on the shear band thickness development. A typical hardening modulus evolution for gouge material is considered and allows to follow the evolution of the localization zone during shearing. We observe a decrease of the shear band 
thickness at the beginning of the localization process, followed by a progressive increase towards a steady value. By assuming an exponential grain size reduction under shearing, which was taken into account by modifying the internal length of the model, it enables to provide a qualitative estimation of the effect grain cataclasis during slip. It is shown that grain communition causes a decrease of the PSZ width.

It is worth emphasizing that the results of the present paper are of qualitative nature. Our target is to explore the tendency of the overall system and the impact of the several physical quantities. However, this investigation is based on LSA, which a has two main drawbacks. The first one is the linearization of the non-linear system of equations, while the second one, is that the linearization is performed to a reference state that does not evolve with deformation. These are strong assumptions for studying the post-bifurcation behavior that have to be explored further numerically. Indeed, in the companion paper (Rattez et al., 2017), the full system of equation is integrated using the Finite Element Method, which allows to also track the exact material response.

\section{References}

An, L. J., Sammis, C. G., 1994. Particle size distribution of cataclastic fault materials from Southern California: A 3-D study. Pure and Applied Geophysics PAGEOPH 143 (1-3), 203-227.

Anthony, J. L., Marone, C. J., 2005. Influence of particle characteristics on granular friction. Journal of Geophysical Research B: Solid Earth 110 (8), $1-14$.

Bauer, E., 2016. Simulation of the Influence of Grain Damage on the Evolution of Shear Strain Localization. In: Continuous Media with Microstructure 2. pp. 231-244.

Ben-Zion, Y., Sammis, C. G., 2003. Characterization of Fault Zones. Pure and Applied Geophysics 160 (3), 677-715. 
Benallal, A., Comi, C., 2003. Perturbation growth and localization in fluidsaturated inelastic porous media under quasi-static loadings. Journal of the Mechanics and Physics of Solids 51 (5), 851-899.

Besdo, D., 1985. Inelastic behaviour of plane frictionless block-systems described as Cosserat media. Archives of Mechanics 37 (6), 603-619.

Biot, M. A., Willis, D. G., 1957. The elastic coefficients of the theory of consolidation. Journal of Applied Mechanics 24, 594-601.

URL http://scholar.google.com/scholar?q=intitle:The+Elastic+ Coefficients+of+the+Theory+of+Consolidation $\{\#\} 0$

Brantut, N., Schubnel, A., Rouzaud, J. N., Brunet, F., Shimamoto, T., 2008. High-velocity frictional properties of a clay-bearing fault gouge and implications for earthquake mechanics. Journal of Geophysical Research: Solid Earth 113 (10).

Brantut, N., Sulem, J., Schubnel, A., 2011. Effect of dehydration reactions on earthquake nucleation: Stable sliding, slow transients, and unstable slip. Journal of Geophysical Research: Solid Earth 116 (5), 1-16.

Cashman, S. M., Baldwin, J. N., Cashman, K. V., Swanson, K., Crawford, R., 2007. Microstructures developed by coseismic and aseismic faulting in nearsurface sediments, San Andres fault, California. Geology 35 (7), 611-614.

Chambon, G., Schmittbuhl, J., Corfdir, A., 2006. Frictional response of a thick gouge sample: 2. Friction law and implications for faults. Journal of Geophysical Research: Solid Earth 111 (9), 1-12.

Chester, F. M., Chester, J. S., 1998. Ultracataclasite structure and friction processes of the Punchbowl fault, San Andreas system, California. Tectonophysics 295 (1-2), 199-221.

Cosserat, E., Cosserat, F., 1909. Theorie des corps deformables. Hermann.

Coussy, O., 2004. Poromechanics. Wiley. 
Cowin, S. C., 1970. Stress Functions for Cosserat Elasticity. International Journal of Solids and Structures 6 (4), 389-398.

de Borst, R., 1991. Simulation of Strain Localization: a Reappraisal of the Cosserat Continuum. Engineering Computations 8 (4), 317-332.

Engelder, J. T., 1974. Cataclasis and the Generation of Fault Gouge. Geological Society of America Bulletin 85 (10), 1515.

Germain, P., 1973. The Method of Virtual Power in Continuum Mechanics. Part 2: Microstructure. SIAM Journal on Applied Mathematics 25 (3), 556-575.

Godio, M., Stefanou, I., Sab, K., Sulem, J., 2016. Multisurface plasticity for Cosserat materials: Plate element implementation and validation. International Journal for Numerical Methods in Engineering 108 (5), 456-484.

URL http://doi.wiley.com/10.1002/nme.5219

Godio, M., Stefanou, I., Sab, K., Sulem, J., Sakji, S., 2017. A limit analysis approach based on Cosserat continuum for the evaluation of the in-plane strength of discrete media: Application to masonry. European Journal of Mechanics, A/Solids 66, 168-192.

URL http://dx.doi.org/10.1016/j.euromechsol.2017.06.011

${ }_{625} \mathrm{Gu}, \mathrm{Y}$., fong Wong, T., 1994. Development of shear localization in simulated quartz gouge: Effect of cumulative slip and gouge particle size. Pure and Applied Geophysics PAGEOPH 143 (1-3), 387-423.

Ikari, M. J., Saffer, D. M., Marone, C. J., 2009. Frictional and hydrologic properties of clay-rich fault gouge. Journal of Geophysical Research: Solid Earth $114(5), 1-18$.

Iordache, M.-M., William, K., 1998. Localized failure analysis in elastoplastic Cosserat continua. Computer Methods in Applied Mechanics and Engineering $151(3-4), 559-586$.

URL http://www.sciencedirect.com/science/article/pii/ 
Kanamori, H., Brodsky, E. E., aug 2004. The physics of earthquakes. Reports on Progress in Physics 67 (8), 1429-1496.

Lachenbruch, A. H., 1980. Frictional Heating, Fluid Pressure, and the Resistance to Fault Motion. Journal of Geophysical Research: Solid Earth 85 (10), 60976112.

Lemaitre, J., Chaboche, J.-L., Benallal, A., Desmorat, R., 2009. Mécanique des matériaux solides-3eme édition. Dunod.

Lyapunov, A. M., 1892. The General Problem of the Stability of Motion (in Russian). Ph.D. thesis, University of Kharkov, Kharkov Mathematical Society.

Manzari, M. T., 2004. Application of micropolar plasticity to post failure analysis in geomechanics. International Journal for Numerical and Analytical Methods in Geomechanics 28 (10), 1011-1032.

Marone, C. J., Scholz, C. H., 1989. Particle-size distribution and microstructures within simulated fault gouge. Journal of Structural Geology 11 (7), 799-814.

Mindlin, R., 1964. Micro-structure in linear elasticity. Archive for Rational Mechanics and Analysis 16 (1).

Montési, L. G. J., Hirth, G., 2003. Grain size evolution and the rheology of ductile shear zones: From laboratory experiments to postseismic creep. Earth and Planetary Science Letters 211 (1-2), 97-110.

Mühlhaus, H.-B., 1986. Scherfugenanalyse bei granularem Material im Rahmen der Cosserat-Theorie. Ingenieur-Archiv 56, 389-399.

Mühlhaus, H.-B., Vardoulakis, I., jan 1987. The thickness of shear bands in granular materials. Géotechnique 37 (3), 271-283.

Muir Wood, D., 2002. Some observations of volumetric instabilities on soils. International Journal of Solids and Structures 39 (13-14), 3429-3449. 
Myers, R., Aydin, A., may 2004. The evolution of faults formed by shearing across joint zones in sandstone. Journal of Structural Geology 26 (5), 947966.

Oda, M., Kazama, H., aug 1998. Microstructure of shear bands and its relation to the mechanisms of dilatancy and failure of dense granular soils. Géotechnique 48 (4), 465-481.

Phillips, N. J., White, J. C., 2017. Grain size-dependent strength of phyllosilicate-rich gouges in the shallow crust: Insights from the SAFOD site. Journal of Geophysical Research: Solid Earth 122 (7), 5789-5812.

URL http://doi.wiley.com/10.1002/2016JB013828

Platt, J. D., Brantut, N., Rice, J. R., 2015. Strain localization driven by thermal decomposition during seismic shear. Journal of Geophysical Research: Solid Earth 120 (6), 4405-4433.

675 Platt, J. D., Rudnicki, J. W., Rice, J. R., may 2014. Stability and localization of rapid shear in fluid-saturated fault gouge: 2. Localized zone width and strength evolution. Journal of Geophysical Research: Solid Earth 119 (5), $4334-4359$.

URL http://doi.wiley.com/10.1002/2013JB010711

Rafini, S., 2008. Comportement hydraulique des milieux faillés. Ph.D. thesis, UQAM.

Rattez, H., Stefanou, I., Sulem, J., Veveakis, M., Poulet, T., 2017. ThermoHydro-Mechanical couplings and strain localisation in 3D continua with microstructure . Part II: Numerical implementation and post-bifurcation analysis. submitted to Journal of Mechanics and Physics of Solids.

Rice, J. R., apr 1975. On the stability of dilatant hardening for saturated rock masses. Journal of Geophysical Research 80 (11), 1531-1536.

URL http://doi.wiley.com/10.1029/JB080i011p01531 
Rice, J. R., 2006. Heating and weakening of faults during earthquake slip. Journal of Geophysical Research: Solid Earth 111 (5).

Rice, J. R., Rudnicki, J. W., Platt, J. D., may 2014. Stability and localization of rapid shear in fluid-saturated fault gouge: 1. Linearized stability analysis. Journal of Geophysical Research: Solid Earth 119 (5), 4311-4333.

URL http://doi.wiley.com/10.1002/2013JB010710

Rudnicki, J. W., 2000. Diffusive instabilities in dilating and compacting geomaterials. Multiscale Deformation and Fracture in Materials and Structures, $159-182$.

Rudnicki, J. W., Rice, J. R., dec 1975. Conditions for the localization of deformation in pressure-sensitive dilatant materials. Journal of the Mechanics and Physics of Solids 23 (6), 371-394.

Sammis, C. G., King, G., Biegel, R. L., 1987. The kinematics of gouge deformation. Pure and Applied Geophysics PAGEOPH 125 (5), 777-812.

URL http://link.springer.com/10.1007/BF00878033

Scholz, C. H., 2002. The mechanics of earthquakes and faulting, second edi Edition. Cambridge.

Scott, D., Marone, C. J., Sammis, C. G., 1994. the apparent friction of granular fault gouge in sheared layers. Journal of Geophysical Research 99 (84), 72317246.

Scuderi, M. M., Niemeijer, A. R., Collettini, C., Marone, C. J., may 2013. Frictional properties and slip stability of active faults within carbonateevaporite sequences: The role of dolomite and anhydrite. Earth and Planetary Science Letters 369-370, 220-232.

Shipton, Z. K., Soden, A., Kirkpatrick, J. D., Bright, A. M., Lunn, R. J., 2006. How thick is a fault? Fault displacement thickness scaling revisited. 
Stefanou, I., Sulem, J., Vardoulakis, I., 2010. Homogenization of interlocking masonry structures using a generalized differential expansion technique. International Journal of Solids and Structures 47 (11), 1522-1536.

Steinmann, P., Willam, K., 1991. Localization within the Framework of Mi296-313.

URL http://dx.doi.org/10.1007/978-3-642-48890-0\{_\}24

Storti, F., Billi, A., Salvini, F., 2003. Particle size distributions in natural carbonate fault rocks : insights for non-self-similar cataclasis 206, 173-186.

740 Sulem, J., Famin, V., 2009. Thermal decomposition of carbonates in fault zones: Slip-weakening and temperature-limiting effects. Journal of Geophysical Research 114 (B3), 1-14. 
Sulem, J., Stefanou, I., 2016. Multiphysics couplings and strain localization in geomaterials. In: ALERT Doctoral School 2016. Modelling of instabilities and bifurcation in Geomechanics. pp. 188-213.

URL http://alertgeomaterials.eu/data/school/2016/ 2016\{_\}ALERT\{_\}schoolbook.pdf

Sulem, J., Stefanou, I., Veveakis, M., feb 2011. Stability analysis of undrained adiabatic shearing of a rock layer with Cosserat microstructure. Granular Matter 13 (3), 261-268.

Sulem, J., Vardoulakis, I., 1990. Bifurcation analysis of the triaxial test on rock specimens. A theoretical model for shape and size effect. Acta mechanica 83 (3-4), 195-212.

Sulem, J., Vardoulakis, I., Ouffroukh, H., Boulon, M., Hans, J., mar 2004. Experimental characterization of the thermo-poro-mechanical properties of the Aegion Fault gouge. Comptes Rendus Geoscience 336 (4-5), 455-466.

Togo, T., Shimamoto, T., 2012. Energy partition for grain crushing in quartz gouge during subseismic to seismic fault motion: An experimental study. Journal of Structural Geology 38, 139-155. URL http://dx.doi.org/10.1016/j.jsg.2011.12.014

Tordesillas, A., Peters, J. F., Gardiner, B. S., aug 2004. Shear band evolution and accumulated microstructural development in Cosserat media. International Journal for Numerical and Analytical Methods in Geomechanics 28 (10), 981-1010.

Tse, S. T., Rice, J. R., 1986. Crustal earthquake instability in relation to the depth variation of frictional slip properties. Journal of Geophysical Research 91 (B9), 9452.

URL http://doi.wiley.com/10.1029/JB091iB09p09452

Unterreiner, P., oct 1994. Contribution à l'étude et à la modélisation numérique 
des sols cloués : application au calcul en déformation des ouvrages de soutènement. Ph.D. thesis, Ecole Nationale des Ponts et Chaussées.

Vardoulakis, I., sep 1985. Stability and bifurcation of undrained, plane rectilinear deformations on water-saturated granular soils. International Journal for Numerical and Analytical Methods in Geomechanics 9 (5), 399-414.

Vardoulakis, I., mar 1986. Dynamic stability analysis of undrained simple shear on water-saturated granular soils. International Journal for Numerical and Analytical Methods in Geomechanics 10 (2), 177-190.

URL http://doi.wiley.com/10.1002/nag.1610100206

780 Vardoulakis, I., 2009. Lecture notes on Cosserat continuum mechanics with application to the mechanics of granular media.

URL http://geolab.mechan.ntua.gr/teaching/lectnotes/CCM2009.pdf

Vardoulakis, I., Sulem, J., 1995. Bifurcation Analysis in Geomechanics. Blackie, Glascow.

${ }_{785}$ Verberne, B. A., Spiers, C. J., Niemeijer, A. R., De Bresser, J. H. P., De Winter, D. A. M., Plümper, O., 2014. Frictional Properties and Microstructure of Calcite-Rich Fault Gouges Sheared at Sub-Seismic Sliding Velocities. Pure and Applied Geophysics 171 (10), 2617-2640.

URL http://link.springer.com/10.1007/s00024-013-0760-0

Veveakis, M., Stefanou, I., Sulem, J., may 2013. Failure in shear bands for granular materials: thermo-hydro-chemo-mechanical effects. Géotechnique Letters 3 (April-June), 31-36.

Viesca, R. C., Garagash, D. I., 2015. Ubiquitous weakening of faults due to thermal pressurization. Nature Geoscience 8 (11), 875-879.

URL http://www. nature.com/doifinder/10.1038/ngeo2554 
Wibberley, C. A., Shimamoto, T., 2003. Internal structure and permeability of major strike-slip fault zones: the Median Tectonic Line in Mie Prefecture, Southwest Japan. Journal of Structural Geology 25 (1), 59-78.

\section{Appendix A. Link between different formulations of the mass balance equation}

After writing the mass conservation for the fluid and the solid phase (see section 3), we obtain equations 2.12 and 2.14.

$$
\begin{array}{r}
-\rho^{s} \frac{\partial n}{\partial t}+(1-n) \frac{\partial \rho^{s}}{\partial t}+\rho^{s}(1-n) V_{i, i}^{s}=0 \\
\frac{n}{\rho^{f}} \frac{\partial \rho^{f}}{\partial t}+\frac{1-n}{\rho^{s}} \frac{\partial \rho^{s}}{\partial t}+V_{i, i}^{s}+\left(n\left(V_{i}^{f}-V_{i}^{s}\right)\right)_{, i}=0
\end{array}
$$

As $V_{i, i}^{s}=\dot{\varepsilon_{v}}$, Eqs. A.1 and A.2 can be written:

$$
\begin{array}{r}
-\frac{1}{1-n} \frac{\partial n}{\partial t}+\frac{1}{\rho^{s}} \frac{\partial \rho^{s}}{\partial t}+\dot{\varepsilon_{v}}=0 \\
\frac{n}{\rho^{f}} \frac{\partial \rho^{f}}{\partial t}+\frac{1-n}{\rho^{s}} \frac{\partial \rho^{s}}{\partial t}+\dot{\varepsilon_{v}}+\left(n\left(V_{i}^{f}-V_{i}^{s}\right)\right)_{, i}=0
\end{array}
$$

$\mathrm{n}$ represents the porosity of the current volume $d \Omega_{t}$, as it refers the current volume to the current configuration, it is called the Eulerian porosity (Coussy, 2004). In contrast, the Lagrangian porosity, written here $\phi$, refers the current porous volume to the initial volume $d \Omega_{t 0}$. We have the relationship:

$$
n d \Omega_{t}=\phi d \Omega_{t 0}
$$

The hypothesis of infinitesimal transformations gives us the approximation:

$$
d \Omega_{t 0} \approx \frac{d \Omega_{t}}{1+\varepsilon_{v}} \approx d \Omega_{t}\left(1-\varepsilon_{v}\right)
$$

If we insert Eq. A.6 in Eq. A.5, take the time derivative and neglect the second order terms, we get the relationship between the variation of Lagrangian porosity and Eulerian porosity. 


$$
\dot{n}=\dot{\phi}-\phi \dot{\varepsilon_{v}}
$$

810

From Eqs. A.3, A.5 and A.7, we have:

$$
\begin{array}{r}
\dot{\varepsilon_{v}}=\dot{\phi}-\frac{1-\phi}{\rho^{s}} \frac{\partial \rho^{s}}{\partial t} \\
\frac{\phi}{\rho^{f}} \frac{\partial \rho^{f}}{\partial t}+\frac{1-\phi}{\rho^{s}} \frac{\partial \rho^{s}}{\partial t}+\dot{\varepsilon_{v}}+\left(\phi\left(V_{i}^{f}-V_{i}^{s}\right)\right)_{, i}=0
\end{array}
$$

We can insert the expression of $\varepsilon_{v}$ in A.8 into A.9.

$$
\frac{\phi}{\rho^{f}} \frac{\partial \rho^{f}}{\partial t}+\dot{\phi}+\left(\phi\left(V_{i}^{f}-V_{i}^{s}\right)\right)_{, i}=0
$$

Following Rice (2006), the porosity is then decomposed into its elastic and inelastic part $n=n^{e l}+n^{i e}$. Thus,

$$
\frac{\partial \phi}{\partial t}=\frac{\partial \phi^{e l}}{\partial t}+\frac{\partial \phi^{i e}}{\partial t}=\phi \beta^{n} \frac{\partial p}{\partial t}+\phi \lambda^{n} \frac{\partial T}{\partial t}+\frac{\partial \phi^{i e}}{\partial t}
$$

where $\beta^{n}$ and $\lambda^{n}$ are the compressibility and the thermal expansivity of the pore volume respectively.

$$
\begin{array}{r}
\beta^{n}=\frac{\beta^{d}-\beta^{s}}{\phi}-\beta^{s} \\
\lambda^{n}=\lambda^{s}
\end{array}
$$

where $\beta^{d}=1 / K$ is the drained compressibility. Finally, from Eqs.A.11, A.10, 2.15 and 2.16

$$
\frac{\partial \mathrm{p}}{\partial t}=c_{h y}^{*} p_{, i i}+\Lambda \frac{\partial \mathrm{T}}{\partial t}-\frac{1}{\beta^{* *}} \frac{\partial \phi^{i e}}{\partial t}
$$

In Eq.A.14, $c_{h y}^{*}=\chi /\left(\eta^{f} \beta^{* *}\right)$ is the hydraulic diffusivity, $\beta^{* *}=\phi\left(\beta^{f}+\beta^{n}\right)$ is the storage capacity, $\Lambda=\left(\lambda^{f}-\lambda^{n}\right) /\left(\beta^{f}+\beta^{n}\right)$ is the undrained thermal 820 pressurization coefficient (Rice, 2006). 


\section{Appendix B. Micro-mechanical considerations}

In this section, we will follow the method presented in Mühlhaus and Vardoulakis (1987) to define the stress and plastic strain invariants for a granular medium modelled by a Cosserat continuum. The granular medium is represented by a random assembly of spheres with a radius $R$. $R$ represents an effective radius of the grains. As granular materials are composed of grains with different shapes and sizes, it is usually assumed to be the mean grain size for granular materials with a narrow grain size distribution. Which length would be appropriate for a larger grain size distribution is actually an open question. As the Cosserat material length controls the width of the strain localization zone, this parameter can be back analyzed from field or laboratory experiments. For zero-entropy materials such as monodisperse blocky structures, the appropriate Cosserat length is found to be the block size (Besdo, 1985, Godio et al., 2017). If we consider two spheres in contact embedded in a continuum, which is defined by the velocity and spin fields $v_{i}$ and $\dot{\omega}_{i}^{c}$, like in Fig. B.16, the relative velocity and spin velocity of the 2 particles are given by (for small rotations):

$$
\begin{array}{r}
v_{i}^{*}-v_{i}=2 R v_{i, j} n_{j} \\
\dot{\omega}_{i j}^{c *}-\dot{\omega}_{i j}^{c}=2 R \dot{\omega}_{i j, k}^{c} n_{k}
\end{array}
$$

(.) denotes the time derivative. $\dot{\omega}_{i}^{c}$ is linked to the micro-deformation tensor (or Cosserat rotation tensor) by $\dot{\omega}_{i j}^{c}=-e_{i j k} \dot{\omega}_{k}^{c}$ ( $e_{i j k}$ is the Levi-Civita tensor).

We express the velocity of the different spheres at the contact point:

$$
\begin{gathered}
v_{i}^{(1)}=v_{i}+R \dot{\omega}_{i j}^{c} n_{j} \\
v_{i}^{(2)}=v_{i}^{*}-R \dot{\omega}_{i j}^{c *} n_{j}
\end{gathered}
$$

840 Thus the relative displacement at the contact point can be written:

$$
\Delta v_{i}=v_{i}^{(2)}-v_{i}^{(1)}=2 R\left(v_{i, j}-\dot{\omega}_{i j}^{c}-R \dot{\omega}_{i j, k}^{c} n_{k}\right) n_{j}
$$




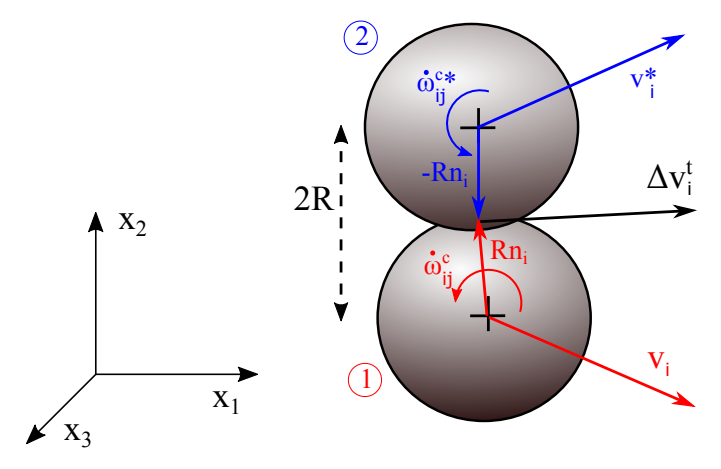

Figure B.16: Relative velocities and spins for 2 grains in contact

or,

$$
\Delta v_{i}=2 R\left(\dot{\gamma}_{i j}+R e_{i j k} \dot{\kappa}_{k l} n_{l}\right) n_{j}
$$

$\dot{\gamma}_{i j}$ is the strain rate tensor and $\dot{\kappa}_{i j}$, the curvature rate tensor. The tangential part, of the relative velocity is:

$$
\Delta v_{i}^{t}=2 R\left(\dot{\gamma}_{i j}-\dot{\gamma}_{p q} n_{p} n_{q} \delta_{i j}+R e_{i j k} \dot{\kappa}_{k l} n_{l}\right) n_{j}
$$

We can calculate its quadratic mean around a sphere of radius $R$, which defines a invariant measure of slip rate:

$$
<\Delta v^{t}>=\left[\frac{1}{S} \int_{s} \Delta v_{i}^{t} \Delta v_{i}^{t} d S\right]^{1 / 2}
$$

where $S$ is the surface of the sphere. By using the following identities:

$$
\begin{array}{r}
\int_{s} n_{i} n_{j} d S=\frac{4 \pi}{3} R^{2} \delta_{i j} \\
\int_{s} n_{i} n_{j} n_{k} d S=0 \\
\int_{s} n_{i} n_{j} n_{k} n_{l} d S=\frac{4 \pi}{15} R^{2}\left(\delta_{i j} \delta_{k l}+\delta_{i k} \delta_{j l}+\delta_{i l} \delta_{j k}\right)
\end{array}
$$

we get,

$$
<\Delta v^{t}>=\sqrt{\frac{16}{15} \dot{e}_{i j} \dot{e}_{i j}-\frac{4}{15} \dot{e}_{i j} \dot{e}_{j i}+R^{2}\left(\frac{16}{15} \dot{k}_{i j} \dot{k}_{i j}-\frac{4}{15} \dot{k}_{i j} \dot{k}_{j i}\right)}
$$


$\dot{e}_{i j}$ and $\dot{k}_{i j}$ are the deviatoric part of the strain rate tensor and curvature rate tensor respectively.

In order to retrieve the classical shear strain rate intensity $\sqrt{2 \dot{e}_{i j} \dot{e}_{i j}}$ when 845 the Cosserat effects vanishes $\left(\dot{e}_{i j}=\dot{e}_{j i}\right.$ and $\left.\dot{\kappa}_{i j} \rightarrow 0\right)$, the coefficients in Eq. B.8 are multiplied by a factor $5 / 2$ (Vardoulakis, 2009). It results in the following definition for $\dot{\gamma}^{p}$ :

$$
\begin{array}{r}
\dot{\gamma}^{p}=\sqrt{g_{1} \dot{e}_{i j} \dot{e}_{i j}+g_{2} \dot{e}_{i j} \dot{e}_{j i}+R^{2}\left(g_{3} \dot{k}_{i j} \dot{k}_{i j}+g_{4} \dot{k}_{i j} \dot{k}_{j i}\right)} \\
g_{1}=g_{3}=\frac{8}{3} \quad \text { and } \quad g_{2}=g_{4}=-\frac{2}{3}
\end{array}
$$

The expression of the shearing intensity $\tau$ that is dual in energy with $\dot{\gamma}^{p}$ is deduced from Eq. B.9. We set the plastic strain rate to be equal to the Lagrange multiplier and we obtain the values of the coefficients in $\tau$ by identification of the different terms on the two sides of this equality. Its final form is:

$$
\begin{array}{r}
\tau=\sqrt{h_{1} s_{i j} s_{i j}+h_{2} s_{i j} s_{j i}+\frac{1}{R^{2}}\left(h_{3} m_{i j} m_{i j}+h_{4} m_{i j} m_{j i}\right)} \\
h_{1}=h_{3}=\frac{2}{5} \quad \text { and } \quad h_{2}=h_{4}=\frac{1}{10}
\end{array}
$$

A micro-mechanical model could also be developed by considering that the normal stresses acting on a elementary volume aren't uniform and, thus, introduce moments. This hypothesis is justified knowing that continuum with micro-structure presents large gradients of stresses and their variation at the level of micro-structure can't be ignored.

By emphasizing the contact tractions over the periphery of grains, it results on a different definitions of the invariants (Sulem and Vardoulakis, 1990) (Mühlhaus and Vardoulakis, 1987). It is called the "static" Cosserat model. 